\title{
Microbial-Catalyzed Biotransformation of Multifunctional Triterpenoids Derived from Phytonutrients
}

\author{
Syed Adnan Ali Shah ${ }^{1,2, *}$, Huey Ling Tan ${ }^{3, *}$, Sadia Sultan ${ }^{1,2, *}$, \\ Muhammad Afifi Bin Mohd Faridz ${ }^{1,2}$, Mohamad Azlan Bin Mohd Shah ${ }^{1,2}$, \\ Sharifah Nurfazilah ${ }^{1,2}$ and Munawar Hussain ${ }^{4}$
}

1 Faculty of Pharmacy, Universiti Teknologi MARA (UiTM), Puncak Alam Campus, 42300 Bandar Puncak Alam, Selangor Darul Ehsan, Malaysia;

E-Mails: fifa200208@gmail.com (M.A.B.M.F.); benzene301@gmail.com (M.A.B.M.S.); sharifahnurfazilah@hotmail.com (S.N.)

2 Atta-ur-Rahman Institute for Natural Products Discovery (AuRIns), Level 9, FF3, Universiti Teknologi MARA (UiTM), Puncak Alam Campus, 42300 Bandar Puncak Alam, Selangor Darul Ehsan, Malaysia

3 Faculty of Chemical Engineering, Universiti Teknologi MARA (UiTM), 40450 Shah Alam, Selangor Darul Ehsan, Malaysia

4 Department of Basic Sciences, DHA Suffa University, Off, Khayaban-e-Tufail, Phase VII (Extension), DHA, Karachi 75500, Pakistan; E-Mail: mhhej@yahoo.co.in

* Authors to whom correspondence should be addressed;

E-Mails: benzene301@yahoo.com or syedadnan@salam.uitm.edu.my (S.A.A.S.); hueyling@salam.uitm.edu.my (H.L.T.); drsadia@puncakalam.uitm.edu.my (S.S.) Tel.: +603-3258-4616 (S.A.A.S.); +603-5543-6310 (H.L.T.); +603-3258-4614 (S.S.); Fax: $+603-3258-4602$ (S.A.A.S.).

Received: 22 May 2014 / in revised form: 12 June 2014 / Accepted: 26 June 2014 / Published: 7 July 2014

\begin{abstract}
Microbial-catalyzed biotransformations have considerable potential for the generation of an enormous variety of structurally diversified organic compounds, especially natural products with complex structures like triterpenoids. They offer efficient and economical ways to produce semi-synthetic analogues and novel lead molecules. Microorganisms such as bacteria and fungi could catalyze chemo-, regio- and stereospecific hydroxylations of diverse triterpenoid substrates that are extremely difficult to produce by chemical routes. During recent years, considerable research has been performed on the microbial transformation of bioactive triterpenoids, in order to obtain biologically active
\end{abstract}


molecules with diverse structures features. This article reviews the microbial modifications of tetranortriterpenoids, tetracyclic triterpenoids and pentacyclic triterpenoids.

Keywords: microbial transformation; tetranortriterpenoids; tetracyclic triterpenoids; pentacyclic triterpenoids; biocatalysis

\section{Introduction}

Natural products extracted from plants, marine sources and microorganisms constitute a rich source of diverse scaffolds for drug discovery. Often they form the backbone of innovative drug discovery programs. They can either be directly used as drugs to treat various diseases, or used as a valuable starting material ("lead") for drug discovery process. From the 1940 s until 2010, 65\% of antibacterial and $41 \%$ of anticancer small molecule drugs developed were either natural products or semi-synthetic derivatives of natural products [1-4]. Structural diversification of multifunctional natural products is often required to improve their solubility, reduce toxicity, or enhance efficacy. Chemical conversions may provide abundant products, but are limited by regio- and stereoselectivity constraints. Moreover, multi-step chemical reactions often result in low overall yield of the final products. However, biocatalytic reactions are well-established "green" techniques for carrying out high chemo-, regio- and stereoselective functionalization of various sensitive and complex molecules under mild reaction conditions and hence are much more attractive for drug development process [5-8]. Biocatalysis, using multi-enzyme systems of fungi, bacteria, and cultured plant suspension cells has the advantage of producing compounds with high selectivity and efficiency under mild conditions. Therefore, biological systems are widely used in the pharmaceutical industry [9-21]. The use of microorganisms such as bacteria and fungi as a biocatalytic system imitates the mamalian metabolism to perform selective transformation reactions and improve the economically and ecologically friendly microbial transformations [22-24]. A number of filamentous fungi are known to perform complex biotransformations that are difficult to achieve by chemical means [22-26]. Many microorganisms, especially certain filamentous fungi, have the ability to transform terpenoids chemo-, regio- and stereoselectively. The fungal-mediated oxidation of terpene under mild conditions appears as an attractive alternative as compared to the traditional chemical methods, have an elevated chemo-, regioand enantioselectivity, and do not generate toxic waste products, and the products obtained can be labeled as "natural" source [5]. Microbial cell-mediated transformations have been extensively used for in vitro in vitro drug metabolic studies. Moreover, microbial transformations can also provide better yields of the metabolites with high selectivity for toxicological and biological studies [22]. Fungi also provide additional advantage in performing reactions similar to mammalian transformations [24].

Triterpenes are plant-derived natural compounds built-up from six isoprene units $\left(\mathrm{C}_{5} \mathrm{H}_{8}\right)$, while triterpenoids consist of both the basic triterpene skeleton and their derivatives that contain oxygen moiety. The simplest triterpene with skeletal structure that forms basis for complex triterpenoids is squalene $\left(\mathrm{C}_{30}\right)$ [8]. It forms the precursor for a structurally diverse group of natural products that display nearly 200 distinct skeletons. These products have been studied for their antiviral (anti-HIV), antineoplastic, anti-inflammatory, anti-ulcerogenic, antimicrobial, anti-plasmodial, hepato- 
and cardio-protective, analgesic, anti-mycotic, and immunomodulatory effects [1,2,6-8]. They are routinely found in numerous medicinal plants. They are also excellent starting material for the synthesis of many fine chemicals due to their homogeneous carbon skeleton [1]. Microbial cell-based transformations of triterpenoids have been developed primarily in the past two decades to produce novel lead molecules, new pharmaceuticals, and agrochemical compounds [7,8,27-31]. Some synthetic oleanane triterpenoids derived from microbial transformation act as multifunctional drugs that regulate the activity of entire networks [32-36]. Microbial biocatalysis has already been proven as powerful tool in the generation of structural diversity in triterpenoid skeletons for future structure-activity relationship studies [5-8].

Figure 1. Structures of tetranortriterpenoids (1), tetracyclic triterpenoids (2), pentacyclic triterpenoids (3-4) and microbial target positions of substituents.
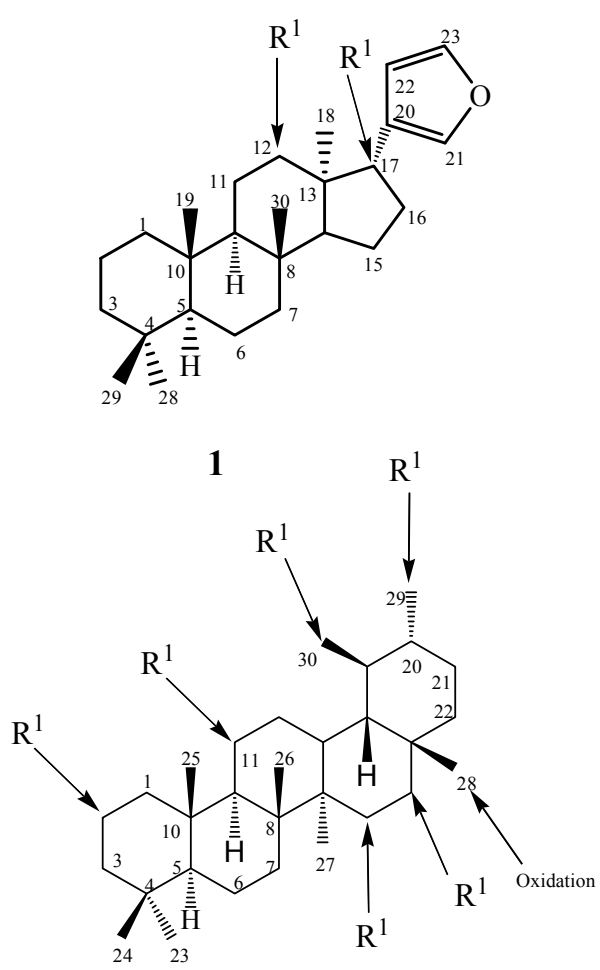

3

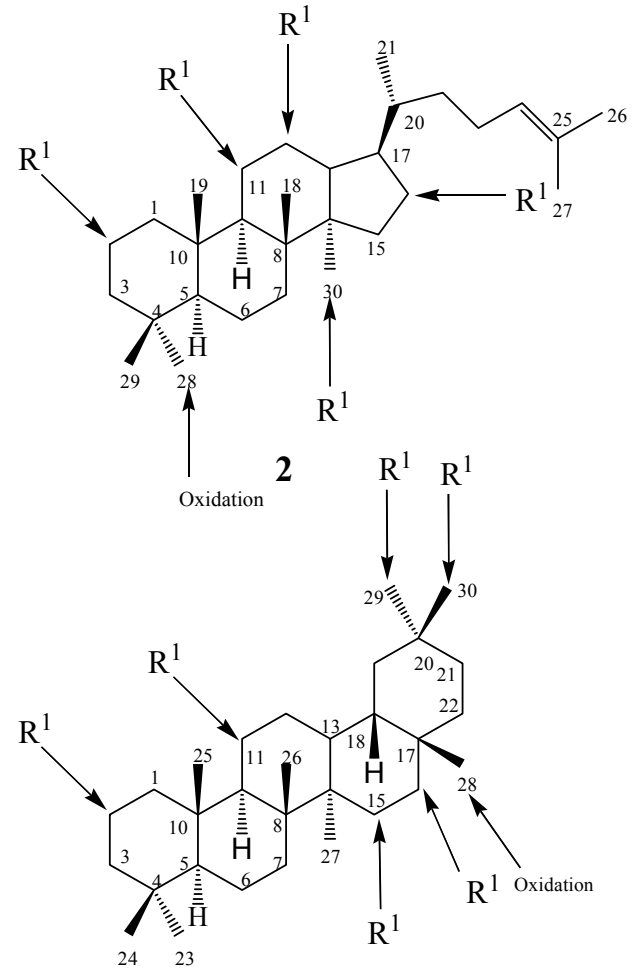

4

This review provides an overview of the structures of diverse and novel products obtained during biotransformation of multifunctional triterpenoid drugs with growing cultures of fungi and bacteria. Different microbial cultures and reaction conditions used in biotransformation of triterpenoid drugs and structure determination methods used in biotransformational processes are discussed.

\section{Fungal Culture Regioselectivity on Triterpenoid Skeleton}

Fungi have been described as useful tool for the biotransformation of natural and semisynthetic triterpenoids $[7,8]$. Nevertheless, in most of the examples, only minor or simple transformations of functional groups have been detected $[6,9]$. 
Microbial cell cultures are capable of performing specific chemical transformations in triterpenoids, such as rearrangement, hydroxylation, oxidation, reduction, hydrolysis, epimerization and isomerization, with high regio- and stereoselectivity as shown in Figure 1 [6-8]. In Figures 2-9, we can see a variation in biocatalytic system introduce regio-selectivity among $12 \beta$ - or 17 $\beta$-hydroxylation in limonoids skeletons [23]. Cunninghamella elegans AS 3.1207 also transforms steroidal saponins into pregnenolones, S. racemosum AS 3.264 converts paeoniflorin into albiflorin [26]. Cunninghamella blakesleeana NRRL 1369 performs complicated rearrangement of tetracyclic triterpenoids into novel ranunculane framework, which reveals the biocatalytic potential of microorganisms in diversification and promoting structural transformation [22-26].

\section{Microbial-Catalyzed Biotransformation of Tetranortriterpenoids}

Limonoids (1), chemically classified as tetranortriterpenoids, are metabolically modified triterpenes having an intact 4,4,8-trimethyl-17-furanylsteroid precursor skeleton (basic limonoid). In some cases, the skeleton is further rearranged and highly oxygenated, creating a structural diversity. They are known to possess anti-cancer, anti-malarial, anti-HIV, antimicrobial and several other pharmacological activities. Azadiradione (5), epoxyazadiradione (14), gedunin (23) and their derivatives fall under this group. The strong antifeedant properties along with anti-plasmoidal, anti-HIV, and anti-inflammatory activities of azadiradione and epoxyazadiradione have attracted the attention of synthetic chemists in the last two decades [23]. In particular, gedunin (23) is a well-studied anti-malarial, anti-carcinogenic and antiulcerogenic agent.

Thulasiram et al. developed a highly efficient fungi-mediated bioconversion for the $12 \beta$ - and $17 \beta$-hydroxylation of the basic limonoid family of compounds (Figure 2). The fungal system belonging to the genera of Mucor (National Collection of Industrial Microorganisms or NCIM, Pune, catalogue no. 881 and abbreviated as M881) efficiently transformed azadiradione (5), epoxyazadiradione (14), gedunin (23) and their derivatives $(\mathbf{8}, \mathbf{1 1}, \mathbf{1 6}, 18$ and 21) into corresponding 12 $\beta$ - and/or $17 \beta$-hydroxy derivatives. These microbial-catalyzed stereo- and regioselective hydroxylation of limonoid skeleton was highly efficient in introducing chemically sensitive functional moieties [23].

Azadiradione (5), a limonoid isolated from the fruit of Azadirachta indica (Neem). The fungus Mucor sp. M881 regioselectively transformed Azadiradione (5), an isolated limonoid from the fruit of Azadirachta indica (Neem), to 17 $\beta$-hydroxyazadiradione (6) and 12 $\beta$-hydroxyazadiradione (7) (Figure 2).

To further investigate the substrate specificity of the organism, the biotransformation was studied with seven natural or semi-synthetic derivatives of azadiradione $(\mathbf{8}, \mathbf{1 1}, \mathbf{1 4}, \mathbf{1 6}, \mathbf{1 8}, 21$ and 23) (see Figures 3-9). Fungi-mediated biocatalysis of 1,2-dihydroazadiradione (8) and 1,2 $\alpha$-epoxyazadiradione (11) resulted in regioselective hydroxylation at $\mathrm{C}-17 \beta$ - and $\mathrm{C}-12 \beta$-sites $(\mathbf{9}, \mathbf{1 0}, \mathbf{1 2}$ and 13) with excellent yields. These biotransformation reactions are shown in Figures 3 and 4. Fermentation of 14, 15ß-epoxyazadiradione (14) and 1,2-dihydroepoxyazadiradione (16) with Mucor sp. M881 (see Figures 5 and 6) afforded regioselective hydroxylation specifically at the C-12 $\beta$ site (15 and 17). The organism also hydroxylated gedunin (23, expanded D ring as six membered lactone) and produced 12ß-hydroxy gedunin (24) as the sole biotransformed product (Figure 9) [23].

Similarly, the Mucor sp. M881 was able to hydroxylate 7-deacetylepoxyazadiradione (21) at the 12ß-position (22) (Figure 8). Nevertheless, when 7-deacetylazadiradione (18, nimbocinol) was used 
as a substrate (Figure 7), this compound was hydroxylated at the 17 -position to produce

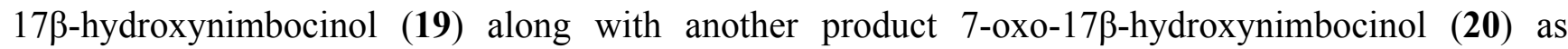
metabolites. These results seems to indicate that the two functional groups (epoxy group in ring D and acetate in ring B) are critical in producing different products (17 $\beta$-hydroxy:12 $\beta$-hydroxy). All these metabolites (6-24) were characterised by detailed ${ }^{1} \mathrm{H}$ and ${ }^{13} \mathrm{C}$ NMR spectroscopy. The location of the hydroxyl functionalities were deduced on the basis of the heteronuclear multiple bond connectivity (HMBC) interactions whereas orientation of $\mathrm{OH}$ groups was deduced on the basis of Nuclear Overhauser Effect spectroscopy (NOESY) correlations [23].

Figure 2. Biotransformation of azadiradione (5) by Mucor sp. M881.
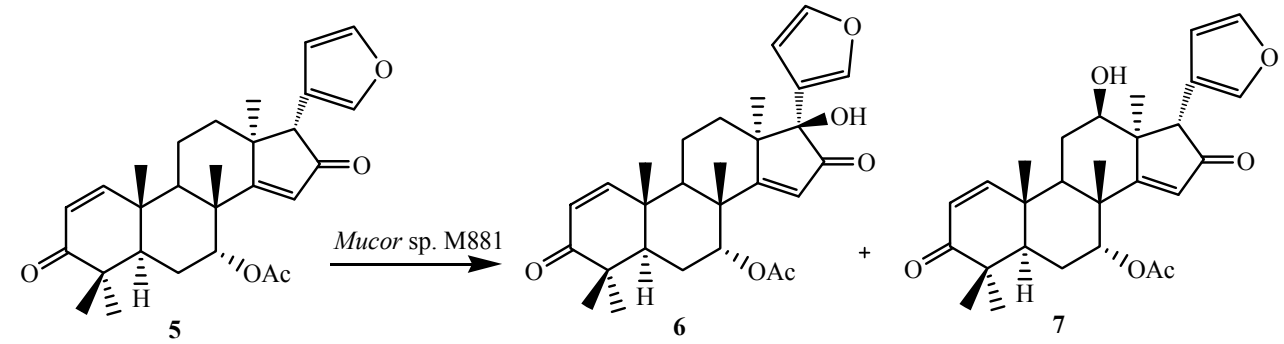

Figure 3. Biotransformation of 1,2-dihydroazadiradione (8) by Mucor sp. M881.

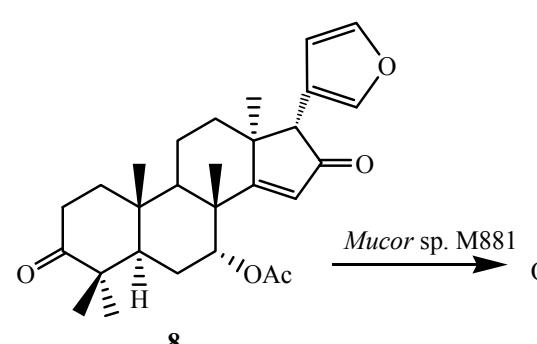

8

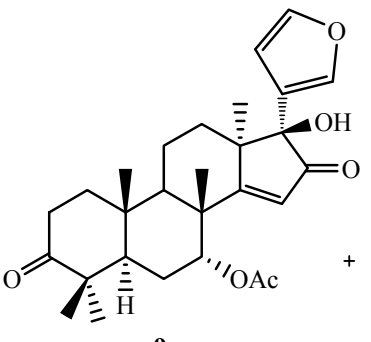

9

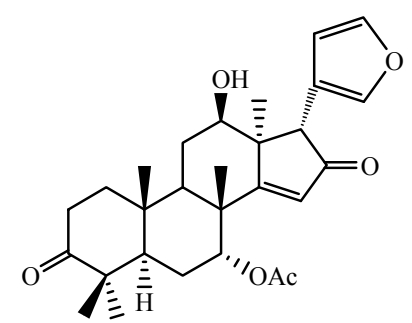

10

Figure 4. Biotransformation of 1,2 $\alpha$-epoxyazadiradione (11) by Mucor sp. M881.
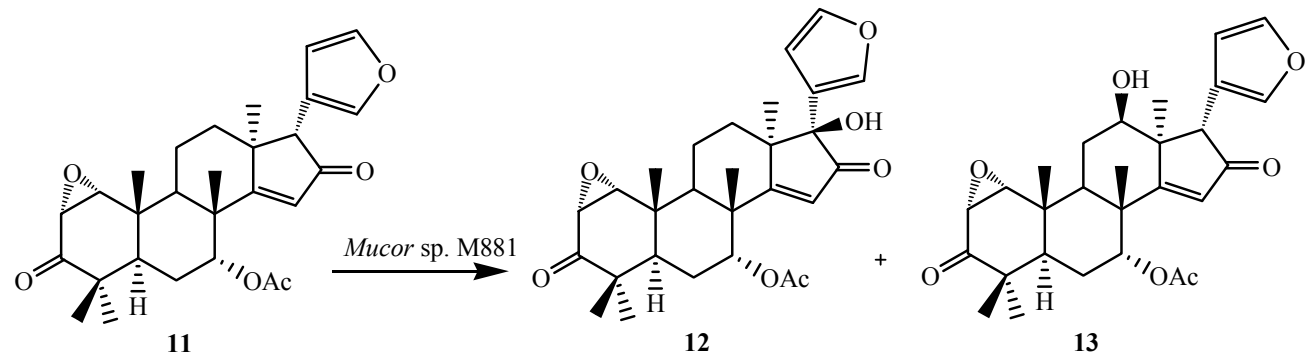

Figure 5. Biotransformation of 14,15ß-epoxyazadiradione (14) by Mucor sp. M881.

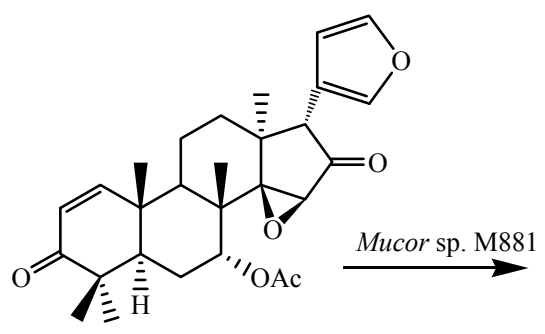

14

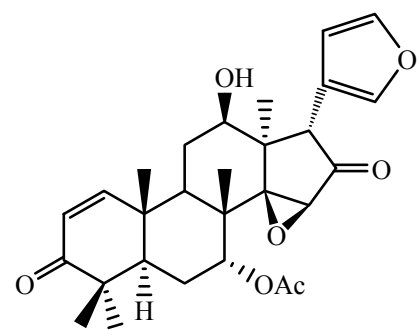

15 
Figure 6. Biotransformation of 1,2-dihydroepoxyazadiradione (16) by Mucor sp. M881.

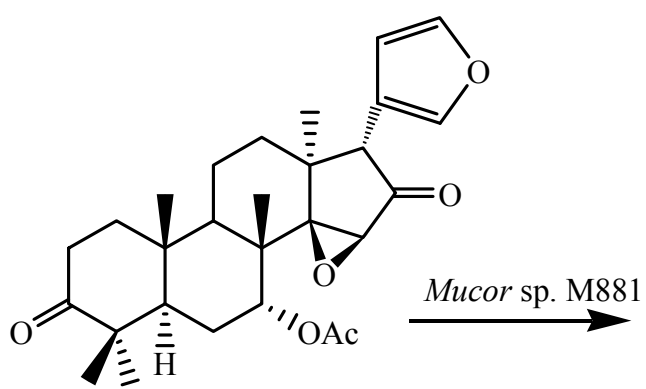

16

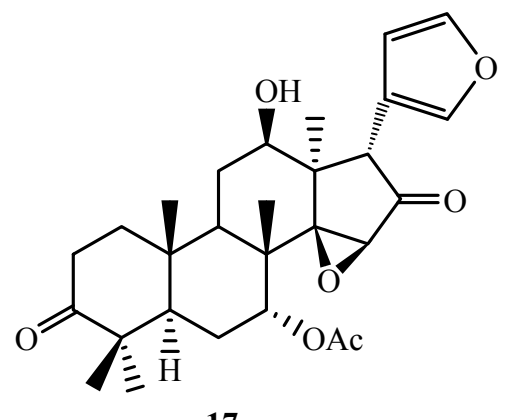

17

Figure 7. Biotransformation of 7-deacetylazadiradione (18) by Mucor sp. M881.

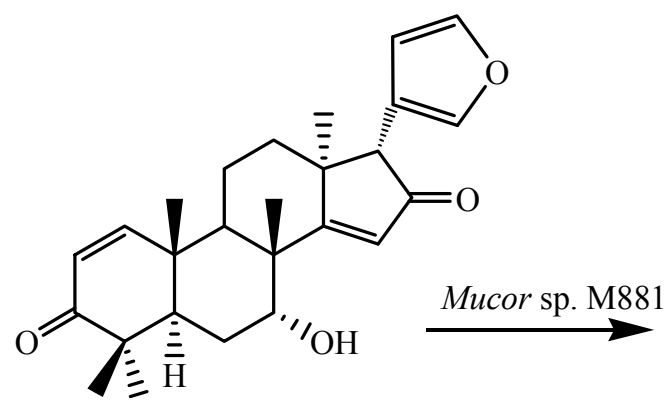

18

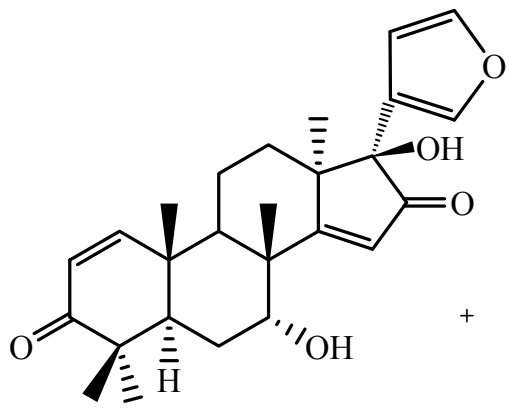

19<smiles>C[C@H]1C(=O)C=C[C@]2(C)C(CC[C@]3(C)C4=CC(=O)[C@@](O)(c5ccoc5)[C@@]4(C)C(=O)C[C@@]32C)[C@]1(C)c1ccoc1</smiles>

20

Figure 8. Biotransformation of 7-deacetylepoxyazadiradione (21) by Mucor sp. M881.

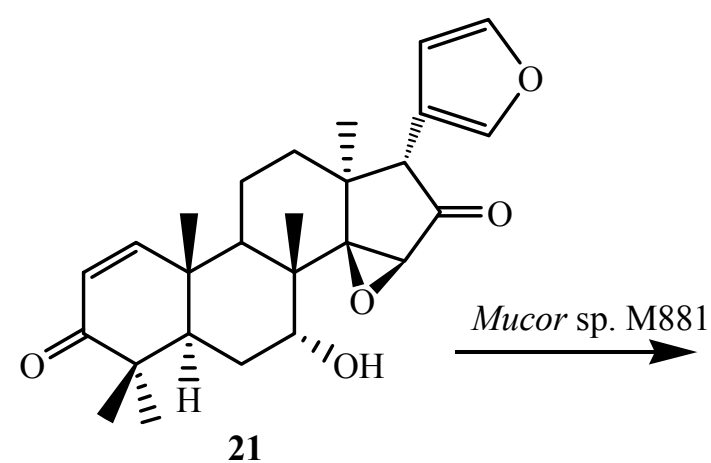

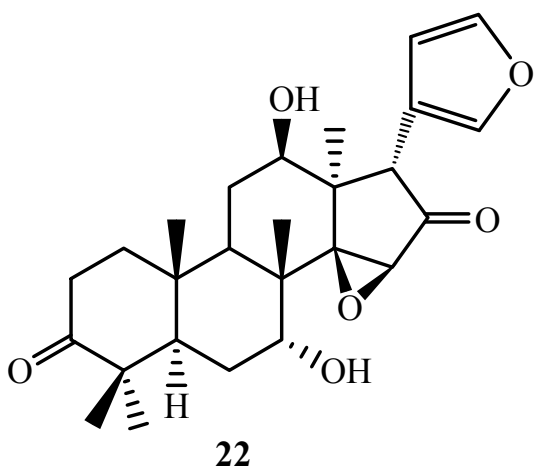

Figure 9. Biotransformation of gedunin (23) by Mucor sp. M881.

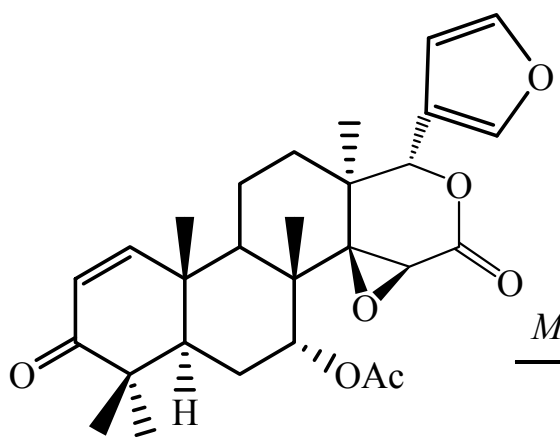

23

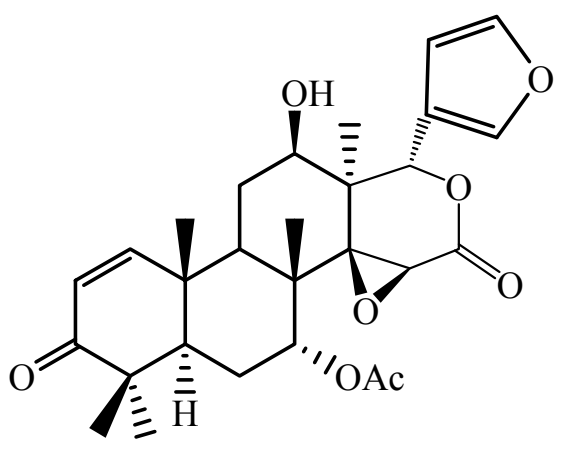

24 


\section{Microbial-Catalyzed Biotransformation of Natural Tetracyclic Triterpenoids}

Cycloastragenol (25), or (20R,24S)-3 $\beta, 6 \alpha, 16 \beta, 25$-tetrahydroxy-20,24-epoxycycloartane (Figure 10), is the genuine sapogenin of astragaloside IV, a major bioactive constituent of Astragalus plants. Astragaloside IV exhibits various pharmacological properties, such as anti-inflammatory, anti-viral, anti-aging and anti-oxidant. It could retard the onset of cellular aging by progressing telomerase activity, up-regulate the immune system by inducing IL-2 release, and elevate the antiviral function of human CD8+ T lymphocytes. Compound $\mathbf{2 5}$ has been considered as a promising new generation of anti-aging agent [26].

The biocatalysis of 25 with two fungal strains, Cunninghamella blakesleeana NRRL 1369 and Glomerella fusarioides ATCC 9552, and the bacterium Mycobacterium sp. NRRL 3805 was investigated by Kuban et al. [24,25]. These strains efficiently transformed $\mathbf{2 5}$ into hydroxylated metabolites along with products formed by cyclization, dehydrogenation and Baeyer-Villiger oxidation resulting in a ring cleavage (Figure 10) [25]. C. blakesleeana efficiently transformed 25 into a complicated rearrangement product, i.e., ring cleavage and methyl group migration of 25, (20R,24S)-3 $\beta, 6 \alpha, 6 \beta, 19,25$-pentahydroxy-ranunculan-9(10)-ene (26) (Figure 10) [24]. With same microorganism, the substrate cycloastragenol (25) underwent several regioselective hydroxylatedproducts,

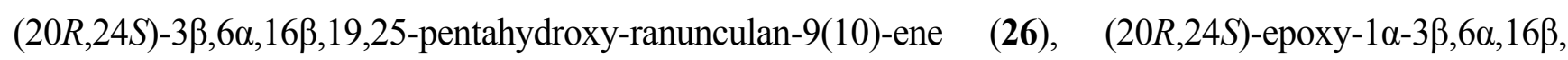
25-pentahydroxycycloartane (27), (20R,24S)-epoxy-3 $\beta, 6 \alpha, 11 \beta, 16 \beta, 25$-pentahydroxycycloartane (28),

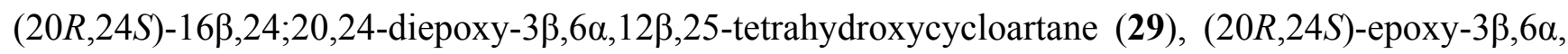

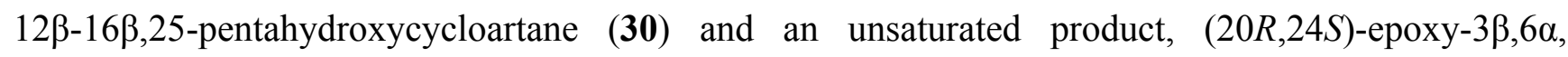
16ß,25-tetrahydroxycycloarta-11(12)-ene (31). Ring cleavage product, 3,4-seco cycloastragenol (32) and

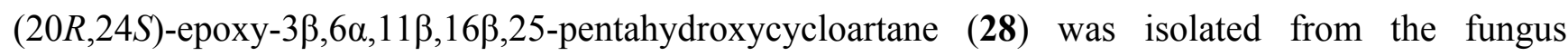
G. fusarioides. The Mycobacterium sp. NRRL 3805 transformed 25 into oxidation product, 33 in $24 \mathrm{~h}$. These biotransformation reactions are shown in Figures 10 and 11 [25].

Ye et al. reported the biotransformation of 25 by Cunninghamella elegans AS 3.1207, Syncephalastrum racemosum AS 3.264 and Doratomyces stemonitis AS 3.1411 [26]. Biocatalytic fermentations of $\mathbf{2 5}$ with $C$. elegans AS 3.1207 for 6 days afforded several regioselective hydroxylated metabolites presented in Figure 12, $(20 R, 24 S)-2 \alpha, 3 \beta, 6 \alpha, 16 \beta, 25$-pentahydroxy-20,24-epoxycycloartane

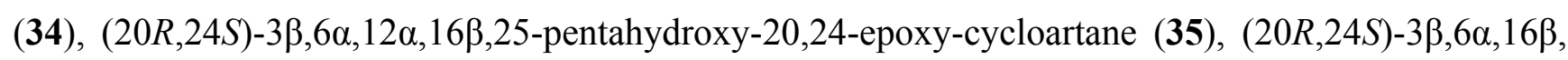
25,28-pentahydroxy-20,24-epoxy-cycloartane $\quad(36), \quad(20 R, 24 S)-3 \beta, 6 \alpha, 16 \beta, 25,29$-pentahydroxy-20, 24-epoxy-cycloartane (37), (20R,24S)-3 $\beta, 6 \alpha, 16 \beta, 25$-tetrahydroxy-20,24-epoxy-cycloartan-28-carbaldehyde

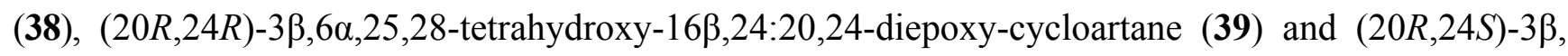
$6 \alpha, 16 \beta, 19,25$-pentahydroxy-ranunculan-9(10)-ene (26) [26]. 
Figure 10. Biotransformation of cycloastragenol (25) by Cunninghamella blakesleeana NRRL 1369.

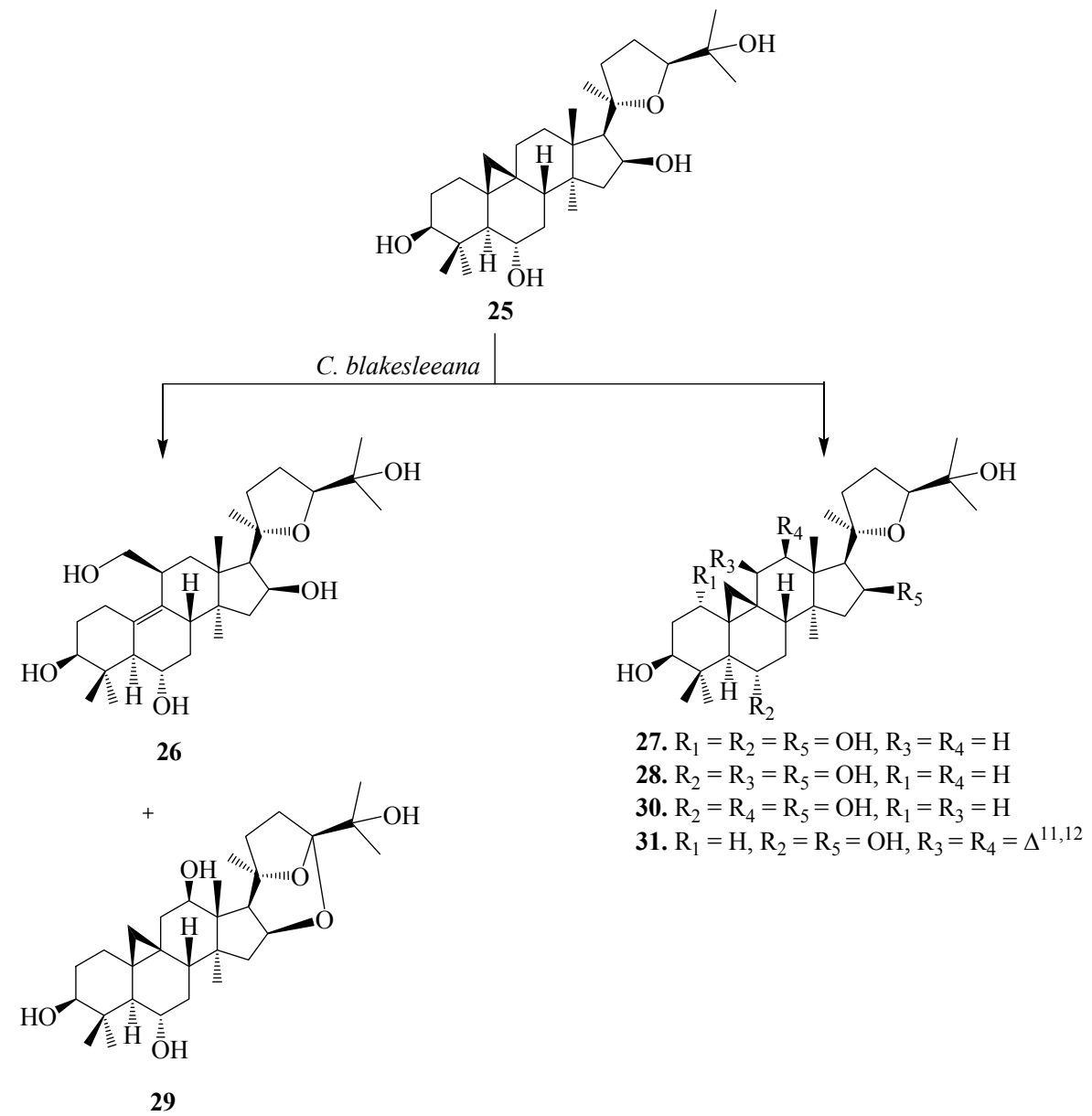

Figure 11. Biocatalytic reactions of Glomerella fusarioides ATCC 9552 and Mycobacterium sp. NRRL 3805 on cycloastragenol (25).

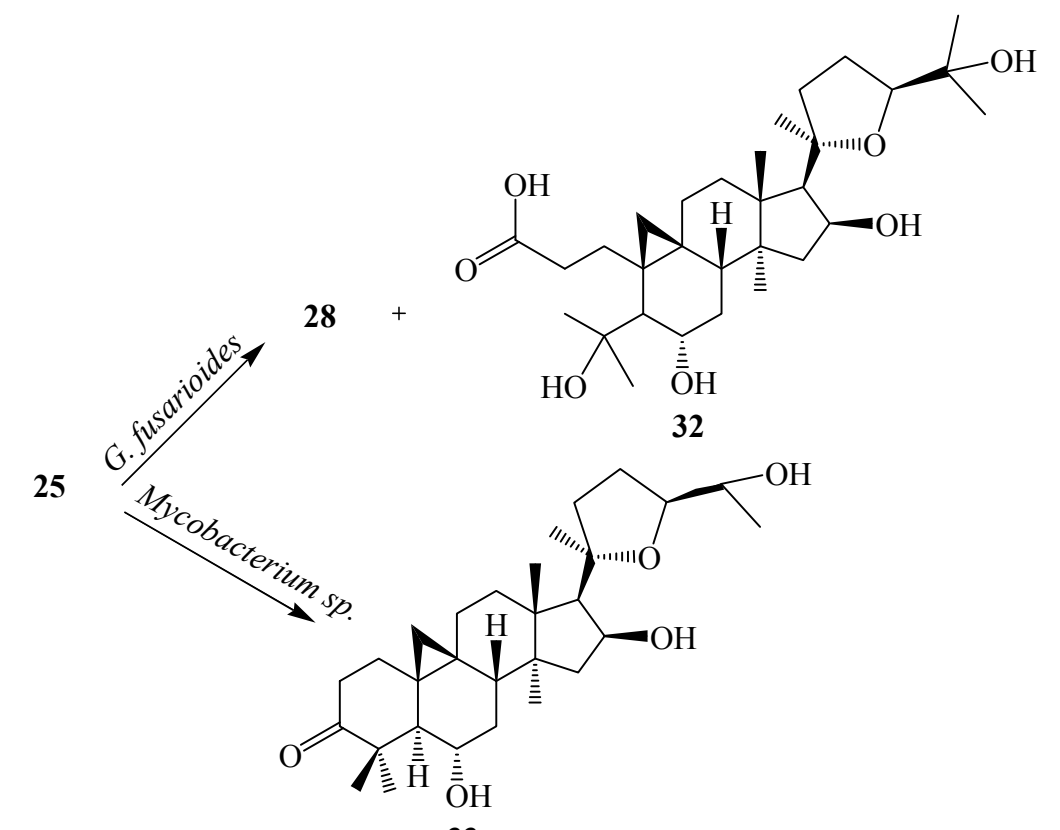


Figure 12. Biocatalytic reactions of Cunninghamella elegans AS 3.1207 on cycloastragenol (25).

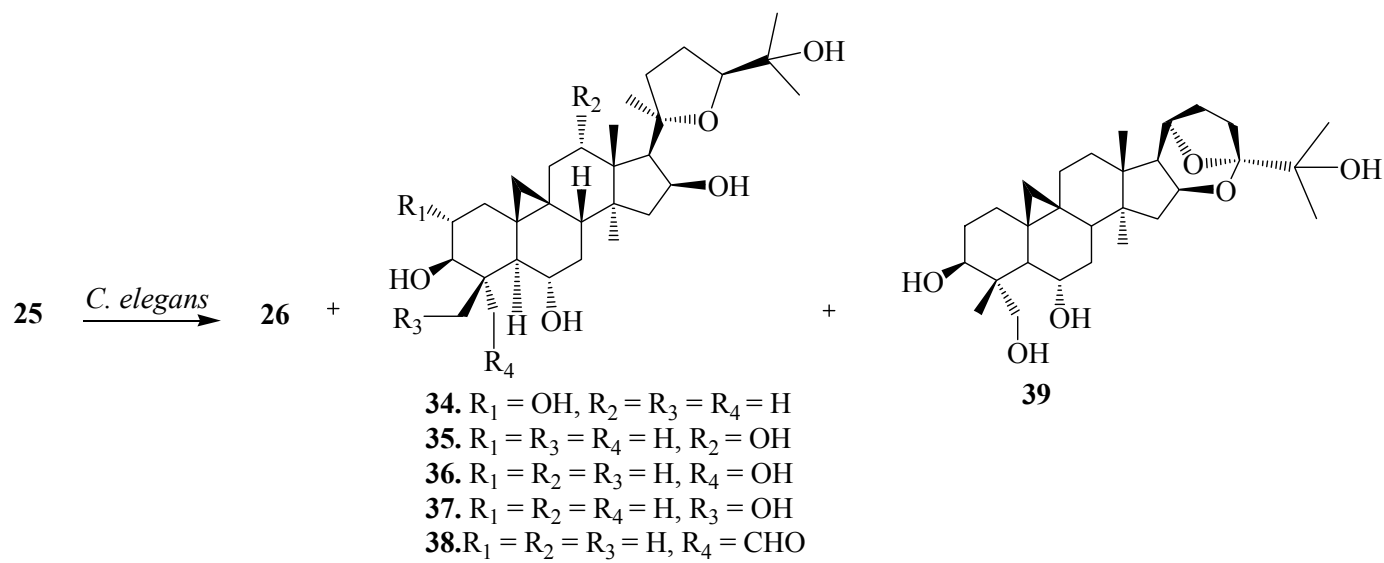

Bioconversion of 25 by $S$. racemosum AS 3.264 yielded (20R,24S)-3 $\beta, 6 \alpha, 16 \beta, 19,25$-pentahydroxyranunculan-9(10)-ene (26), (20R,24S)-3 $\beta, 6 \alpha, 12 \alpha, 16 \beta, 25$-pentahydroxy-20,24-epoxy-cycloartane (35),

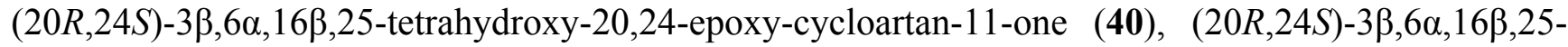
tetrahydroxy-20,24-epoxy-cycloartan-11(12)-ene (41), (20R,24S)-3 $\beta, 6 \alpha, 16 \beta, 25$-tetrahydroxy-19-butoxyranunculan-9(10)-ene (42), (20R,24S)-3 $\beta, 6 \alpha, 16 \beta, 25$-tetrahydroxy-19-isopentenyloxyranunculan-9(10)-ene (43), (20R,24S)-3 $\beta, 6 \alpha, 16 \beta, 25$-tetrahydroxy-19-acetoxy-ranunculan-9(10)-ene (44) and ring expansion metabolite, neoastragenol or $(20 R, 24 S)$-3 $\beta, 6 \alpha, 16 \beta, 25$-tetrahydroxy-20,24-epoxy-9(10)a-homo-19-norcycloartane (45) (Figure 13). D. stemonitis AS 3.1411 transformed 25 to two carbonylated

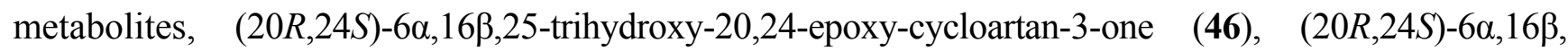
25,30-tetrahydroxy-20,24-epoxy-cycloartan-3-one (47) and 26. These transformations are shown in Figure $14[26]$.

Figure 13. Biotransformation of Syncephalastrum racemosum AS 3.264 on cycloastragenol (25).

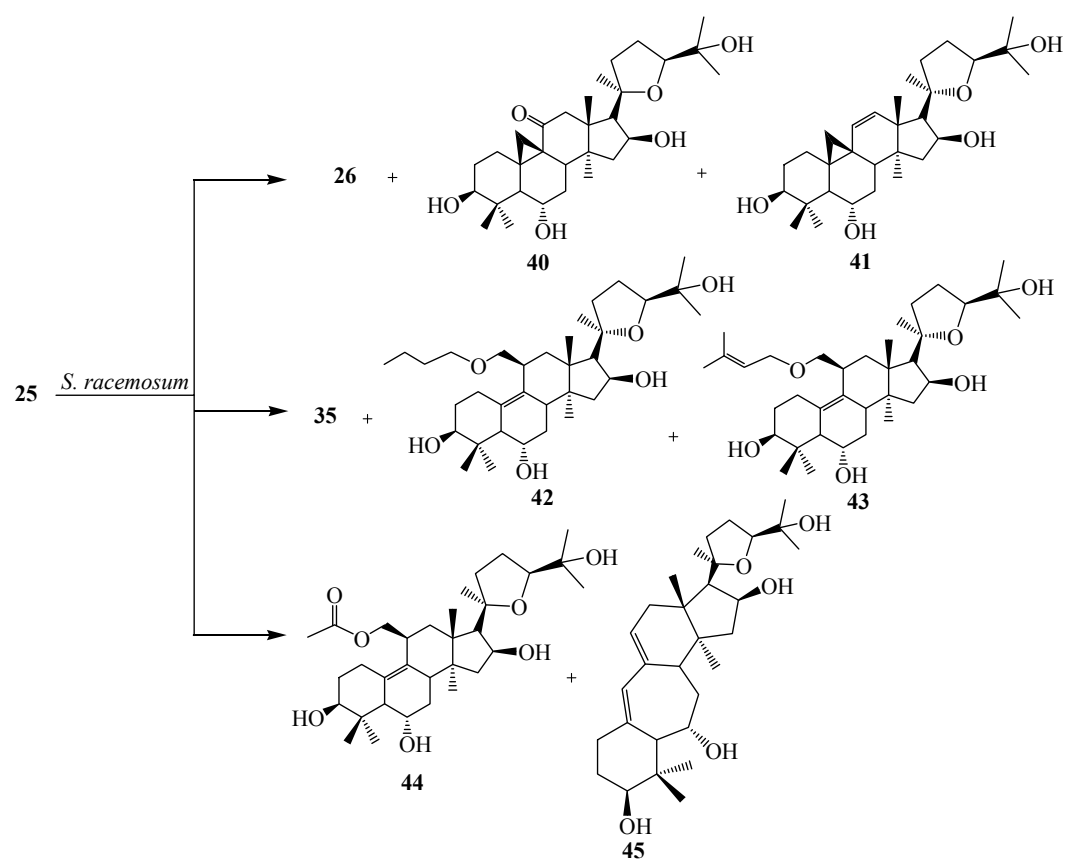


Figure 14. Biotransformation of Doratomyces stemonitis AS 3.1411 on cycloastragenol (25).

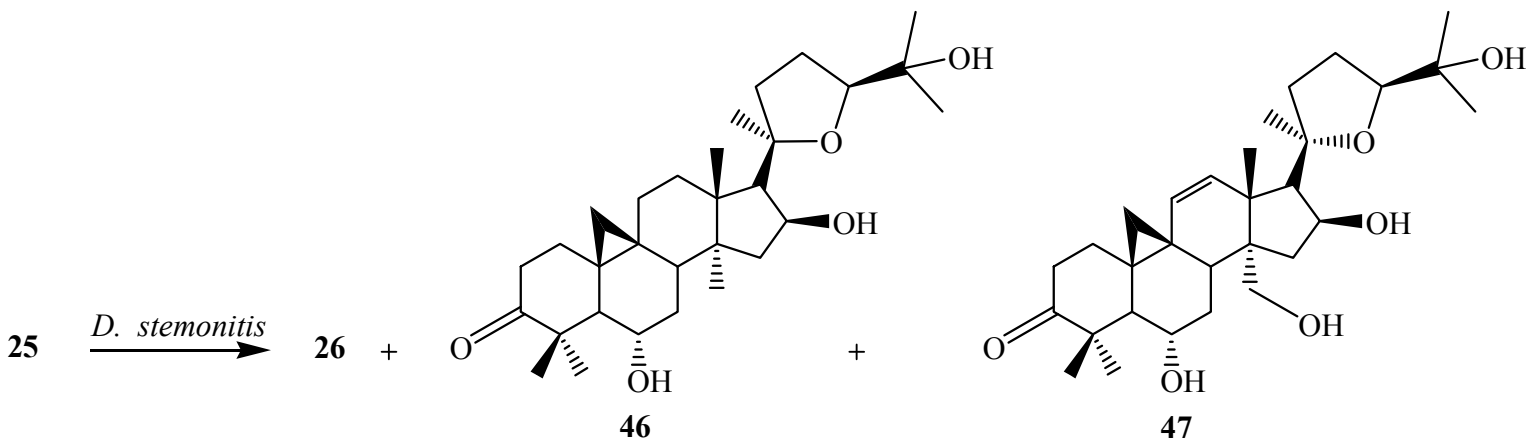

The tetratriterpenoid 20(S)-Protopanaxadiol (48), a glycone of ginsenosides from the Chinese ginseng (Panax ginseng C.A. Mey, Araliaceae) exhibits powerful pleiotropic anti-cancer effects in several cancer cell lines including the inhibition of metastasis. Furthermore, compound $\mathbf{4 8}$ could induce apoptosis through mitochondria mediated apoptotic pathway in Caco-2, U937, THP-1, and SMMC7721 cancer cells [27]. The fungal tansformation of $\mathbf{4 8}$ was reported by Li et al. Fermentation of 48 with Mucor spinosus AS 3.3450 for 5 days yielded eight regioselective hydroxylated metabolites

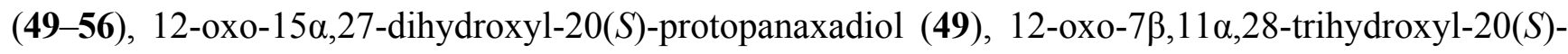

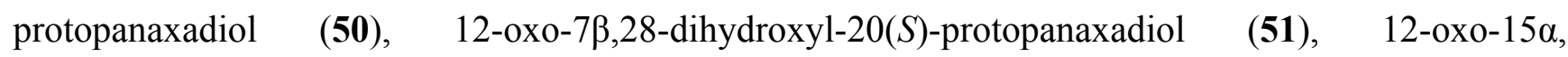
29-dihydroxyl-20(S)-protopanaxadiol (52), 12-oxo-7 $\beta, 15 \alpha$-dihydroxyl-20(S)-protopanaxadiol (53), 12-oxo-7 $\beta, 11 \beta$-dihydroxyl-20(S)-protopanaxadiol (54), 12-oxo-15 $\alpha$-hydroxyl-20(S)-protopanaxadiol (55), and 12-oxo-7 $\beta$-hydroxyl-20(S)-protopanaxadiol (56) (Figure 15). Incubation of 48 with Aspergillus niger AS 3.1858 afforded seven additional hydroxylated metabolites (57-63), 26-hydroxyl-20(S)-protopanaxadiol (57), 23,24-en-25-hydroxyl-20(S)-protopanaxadiol (58), 25,26-en20(S)-protopanaxadiol (59), (E)-20,22-en-25-hydroxyl-20(S)- protopanaxadiol (60), 25,26-en-24(R)hydroxyl-20(S)-protopanaxadiol (61), 25,26-en-24(S)-hydroxyl-20(S)-protopanaxadiol (62) and 23, 24-en-25-ethoxyl-20(S)-protopanaxadiol (63) [28]. These biotransformation reactions are described in Figure 16.

The bacterium Bacillus megaterium metabolized the triterpenoid dipterocarpol (64) to $7 \beta$-hydroxydipterocarpol (65) and 7 $\beta, 11 \alpha$-dihydroxydipterocarpol (66) in $16 \mathrm{~h}$ (Figure 17). The Dipterocarpol (64) and the dihydroxylated product 66 did not displayed cytotoxic activity with HeLa and COS-1 cells while 7 $\beta$-hydroxylated product $\mathbf{6 5}$ exhibited cytotoxicity on both the cell lines [29].

Figure 15. Biotransformation of Mucor spinosus AS 3.3450 on 20(S)-protopanaxadiol (48).

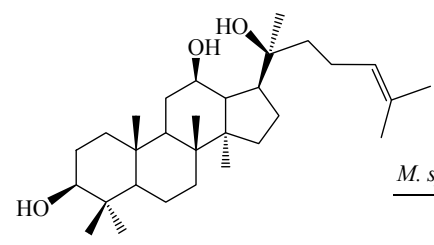

48

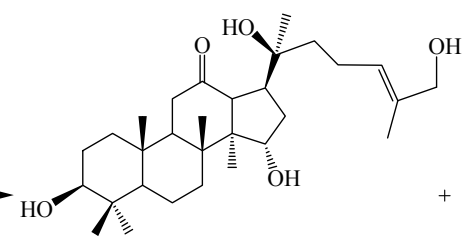

49

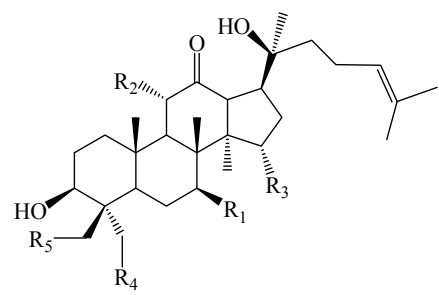

50. $\mathrm{R}_{1}=\mathrm{R}_{2}=\mathrm{R}_{4}=\mathrm{OH}, \mathrm{R}_{3}=\mathrm{R}_{5}=\mathrm{H}$ 51. $\mathrm{R}_{1}=\mathrm{R}_{4}=\mathrm{OH}, \mathrm{R}_{2}=\mathrm{R}_{3}=\mathrm{R}_{5}=\mathrm{H}$ 52. $\mathrm{R}_{1}=\mathrm{R}_{2}=\mathrm{R}_{4}=\mathrm{H}, \mathrm{R}_{3}=\mathrm{R}_{5}=\mathrm{OH}$ 53. $\mathrm{R}_{1}=\mathrm{R}_{3}=\mathrm{OH}, \mathrm{R}_{2}=\mathrm{R}_{4}=\mathrm{R}_{5}=\mathrm{H}$ 54. $\mathrm{R}_{1}=\mathrm{R}_{2}=\mathrm{OH}, \mathrm{R}_{3}=\mathrm{R}_{4}=\mathrm{R}_{5}=\mathrm{H}$ 55. $\mathrm{R}_{1}=\mathrm{R}_{2}=\mathrm{R}_{4}=\mathrm{R}_{5}=\mathrm{H}, \mathrm{R}_{3}=\mathrm{OH}$ 56. $R_{1}=O H, R_{2}=R_{3}=R_{4}=R_{5}=H$ 
Figure 16. Biotransformations of Aspergillus niger AS 3.1858 on 20(S)-protopanaxadiol (48).

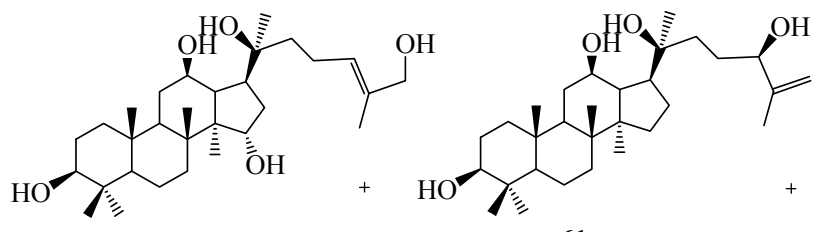

57

61

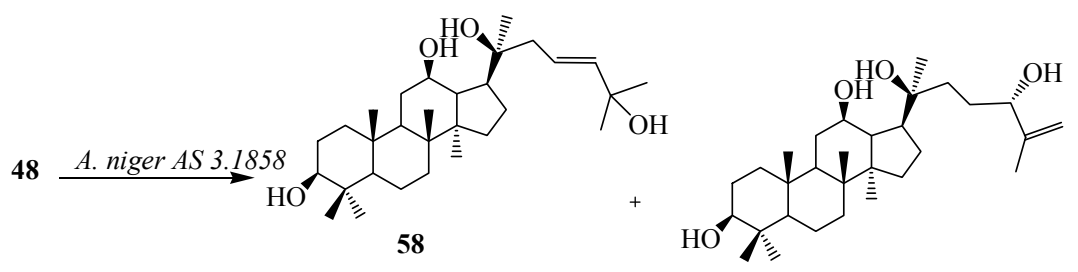

62<smiles>C=C(C)CCC[C@](C)(O)[C@H]1CC[C@]2(C)C1[C@@H](O)CC1[C@@]3(C)CC[C@H](O)C(C)(C)C3CC[C@]12C</smiles>

59<smiles>C/C(=C/CCC(C)(C)O)[C@H]1CC[C@]2(C)[C@H]1C[C@H](O)C[C@@H]1C(C)(C)[C@@H](O)CC[C@]12C</smiles>

63

Figure 17. Biotransformation of dipterocarpol (64) with Bacillus megaterium ATCC 13368.
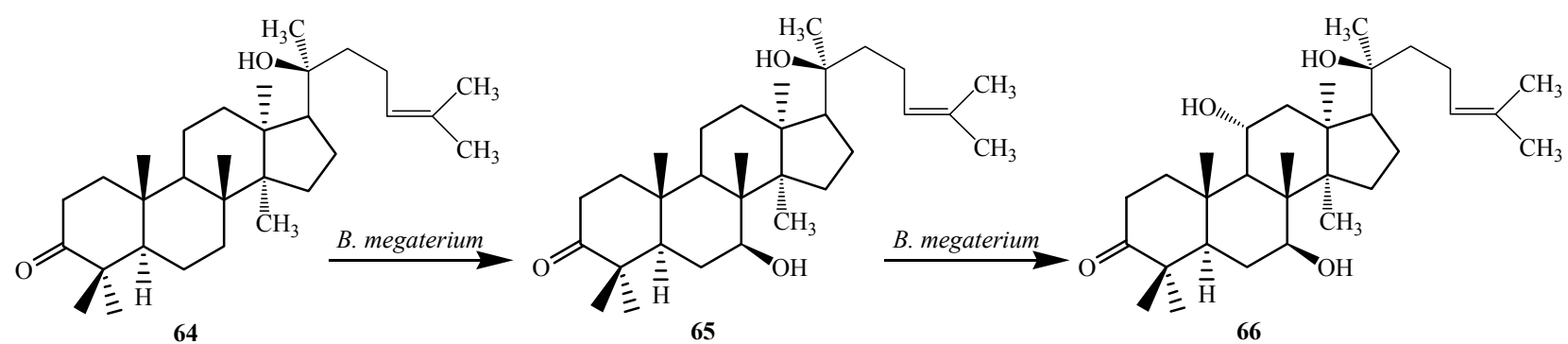

Schisandra propinqua var. sinensis, popularly known as "tie-gu-san" produced by Schisandraceae family in the Shennongjia district of mainland China, is used as folk medicine for the treatment of arthritis, traumatic injury, gastralgia, angeitis, and other related diseases. Nigranoic acid (3,4-secocycloarta- 4(28),24(Z)-diene-3,26-dioic acid, 67) is the first member of class 3,4-secocycloartane triterpenoid produced by Schisandra propinqua, that has been reported to possess a variety of biological activities, including cytotoxic activity toward leukemia and Hela cells, and inhibition of expression of HIV reverse transcriptase and polymerase [31]. Dong et al. studied the microbiological transformation of $\mathbf{6 7}$ by the freshwater fungus Dictyosporium heptasporum YMF1.01213. The organism metabolized 67 into novel nine-membered lactone ring 3,4-secocycloartane triterpenoid

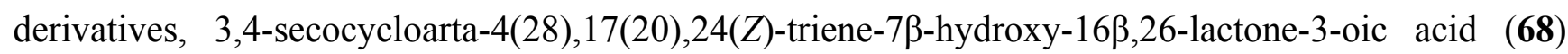

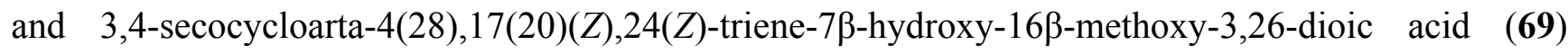
(Figure 18) [30]. 
Dong et al. reported the bioconversion of $\mathbf{6 7}$ by cultures of Trichoderma sp. JY-1. The fungus yielded 15 $\alpha, 16 \alpha$-dihydroxy-3,4-secocyloarta-4(28),17(20),17(E),24(E)-triene-3,26-dioic acid (70) and 16 $\alpha, 20 \alpha$-dihydroxy-18 $(13 \rightarrow 17 \beta)$ abeo-3,4-secocyloarta-4 (28),12(13),24(Z)-triene-3,26-dioic acid (71). Substrate 67 and its transformed products 70 and 71 displayed weak anti-HIV activity with $\mathrm{EC}_{50}$ values of $10.5,8.8$ and $7.6 \mathrm{mg} / \mathrm{mL}$, and therapeutic index values $\left(\mathrm{CC}_{50} / \mathrm{EC}_{50}\right)$ of 8.48, 9.12 and 10.1, respectively (Figure 19) [31].

Figure 18. Microbiological transformation of the triterpene nigranoic acid (67) by the freshwater fungus Dictyosporium heptasporum.

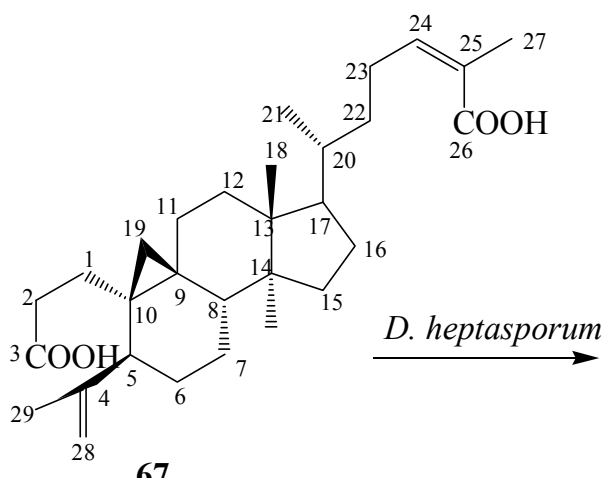

67

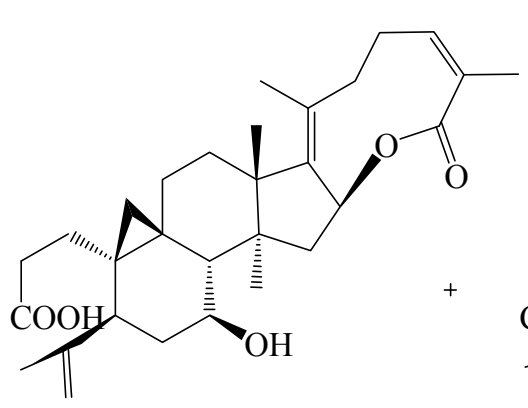

68

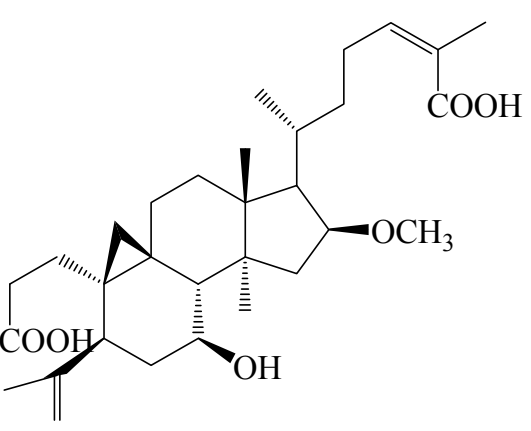

69

Figure 19. Metabolism of 67 by Trichoderma sp. JY-1 culture.

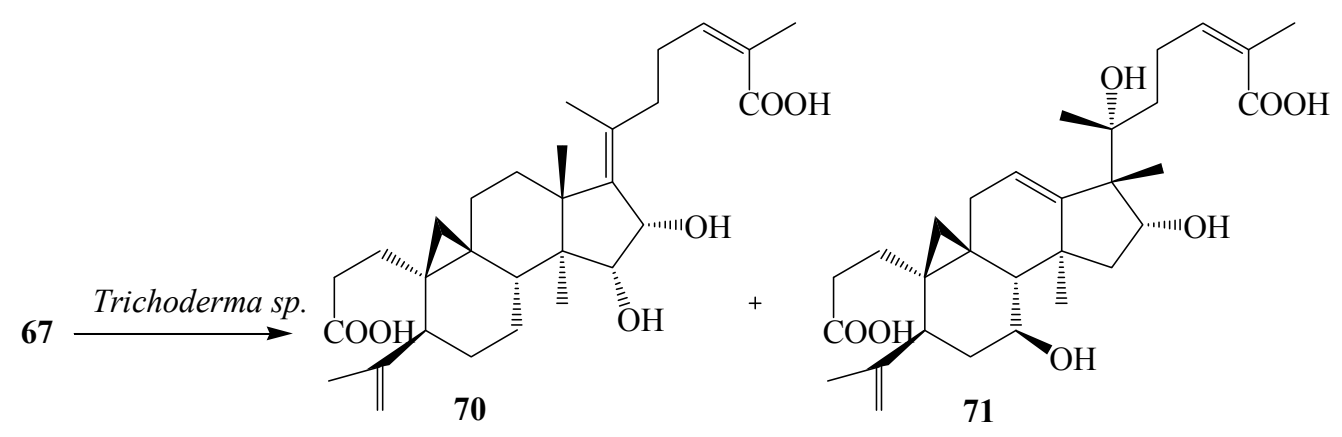

\section{Microbial Transformation of Natural and Semi-Synthetic Pentacyclic Triterpenoids}

Pentacyclic triterpenoids (3-4) are widely distributed in many medicinal plants, such as birch bark (betulin, 153), plane bark (betulinic acid, 154), olive leaves, olive pomace, mistletoe sprouts and clove flowers (oleanolic acid, 72), and apple pomace (ursolic acids, 114). Compounds belonging to this group such as lupane (lupeol, betulin, betulinic acid), oleanane (72 and maslinic acid (73), erythrodiol, $\beta$-amyrin), and ursane (114, uvaol, $\alpha$-amyrin) display various pharmacological effects. These triterpenoids are ideal and potential candidates for designing lead compounds for the development of new bioactive agents [32].

Olean-type pentacyclic triterpenes (OPTs) are plant-derived natural products, abundantly found in many medicinal herbs. They display a remarkable spectrum of biological activities, such as antimalarial, anti-tumor, anti-HIV, anti-microbial, anti-diabetic, and anti-inflammatory activities [32]. The microbial-catalyzed modification of olean-type pentacyclic triterpenes mainly resulted in the substitution of hydroxyl or carbonyl groups to the methyl or methenyl carbons of the skeleton and the 
formation of corresponding glycosides [32]. The presence of such functional moieties, especially at C-3, C-28, and C-30, seems to contribute to the biological activities of pentacyclic triterpenoids $[17,35,36]$.

Oleanolic acid (3ß-hydroxyolean-12-en-28-oic acid, 72) is a natural pentacyclic triterpenoid compounds widely present in the form of free acid or aglycone of triterpenoid saponins. It is usually found in high concentrations in olive-pomace oil [32]. Some oleanolic acids have been reported to be antimalarial, antitumor, hepatoprotective, anti-HIV, and skin protective. They seem to possess $\alpha$-glucosidase inhibitory activity.

Figure 20. Microbial transformation of oleanolic acid (72) by Furarium lini NRRL-68751 and Colletotrichum lini AS3.4486.

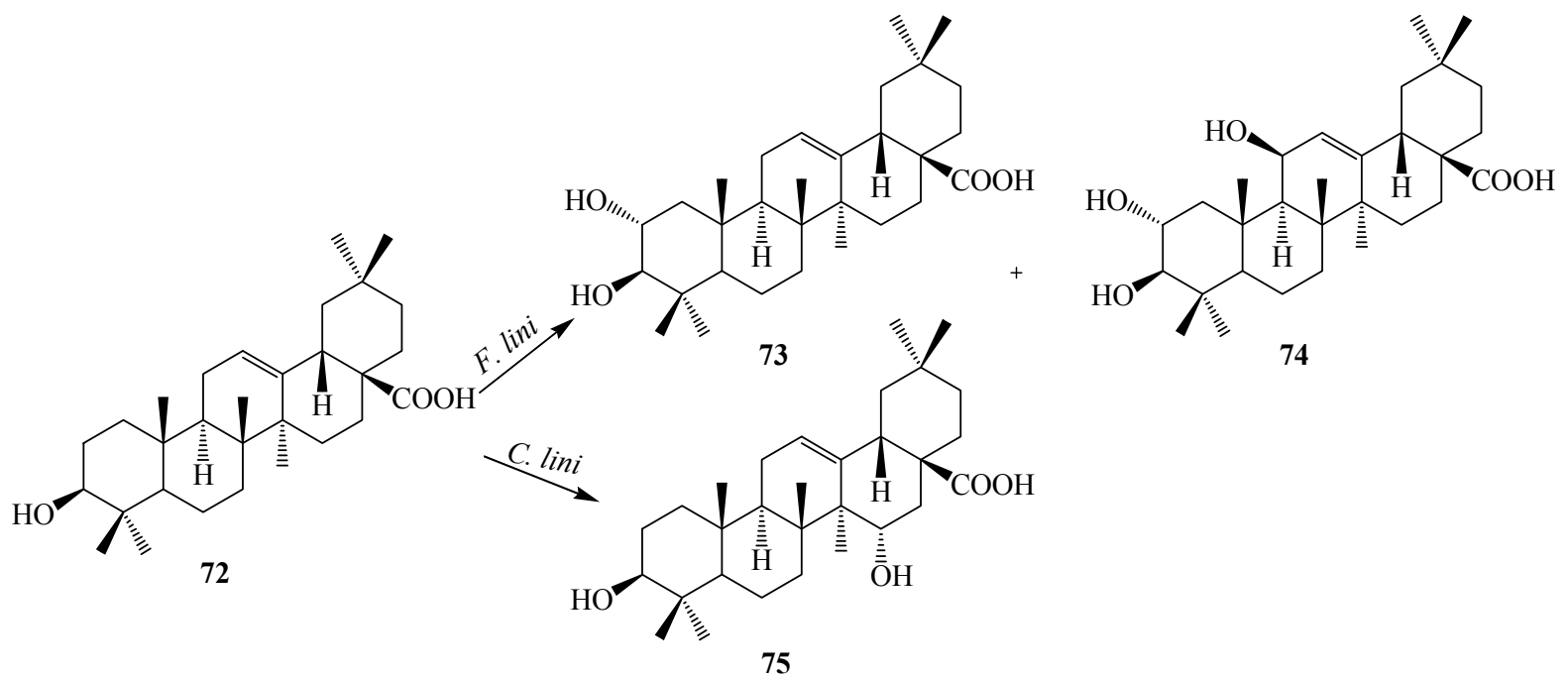

Several biotransformations of oleanolic acid (72) with different microorganisms have been reported so far. Choudhary et al. investigated the metabolism of oleanolic acid (72) with Fusarium lini NRRL-68751 and reported the production of two oxidative metabolites, maslinic acid

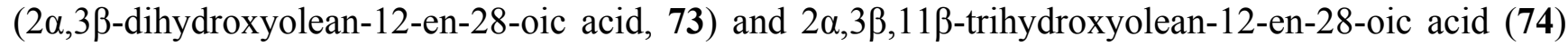
(Figure 20). These metabolites show potent inhibition of $\alpha$-glucosidase activity. Hua et al. reported the metabolism of $\mathbf{7 2}$ with Colletotrichum lini AS3.4486 which resulted in one polar metabolite, 15 $\alpha$-hydroxyl-oleanolic acid (75) (Figure 20) [17,33].

Liu et al. reported microbial transformation of $\mathbf{7 2}$ with Alternaria longipes and Penicillium adametzi. The fungus Alternaria longipes converted $\mathbf{7 2}$ to six different regioselective hydroxylated

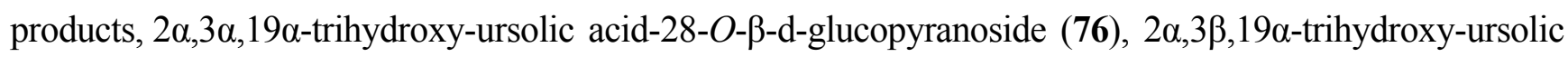
acid-28-O- $\beta$-d-glucopyranoside (77), oleanolic acid 28-O- $\beta$-d-glucopyranosyl ester (78), oleanolic acid-3-O- $\beta$-d-glucopyranoside (79), 3-O-( $\beta$-d-glucopyranosyl)-oleanolic acid-28- $O$ - $\beta$-d-glucopyranoside (80), $2 \alpha, 3 \beta, 19 \alpha$-trihydroxy-oleanolic acid-28-O- $\beta$-d-glucopyranoside (81), while cultures of Penicillium adametzi transformed the $\mathbf{7 2}$ to $21 \beta$-hydroxyl oleanolic acid (82), $7 \alpha, 21 \beta$-dihydroxyl oleanolic acid (83) and 21 $\beta$-hydroxyl oleanolic acid-28-O- $\beta$-d-glucopyranoside (84). These fermentation reactions are presented in Figures 21 and 22. Compounds 79 and 82-84 exhibited stronger cytotoxic activities against Hela cell lines than the substrate 72 [34]. 
Figure 21. Microbial transformation of oleanolic acid (72) by Alternaria longipes.

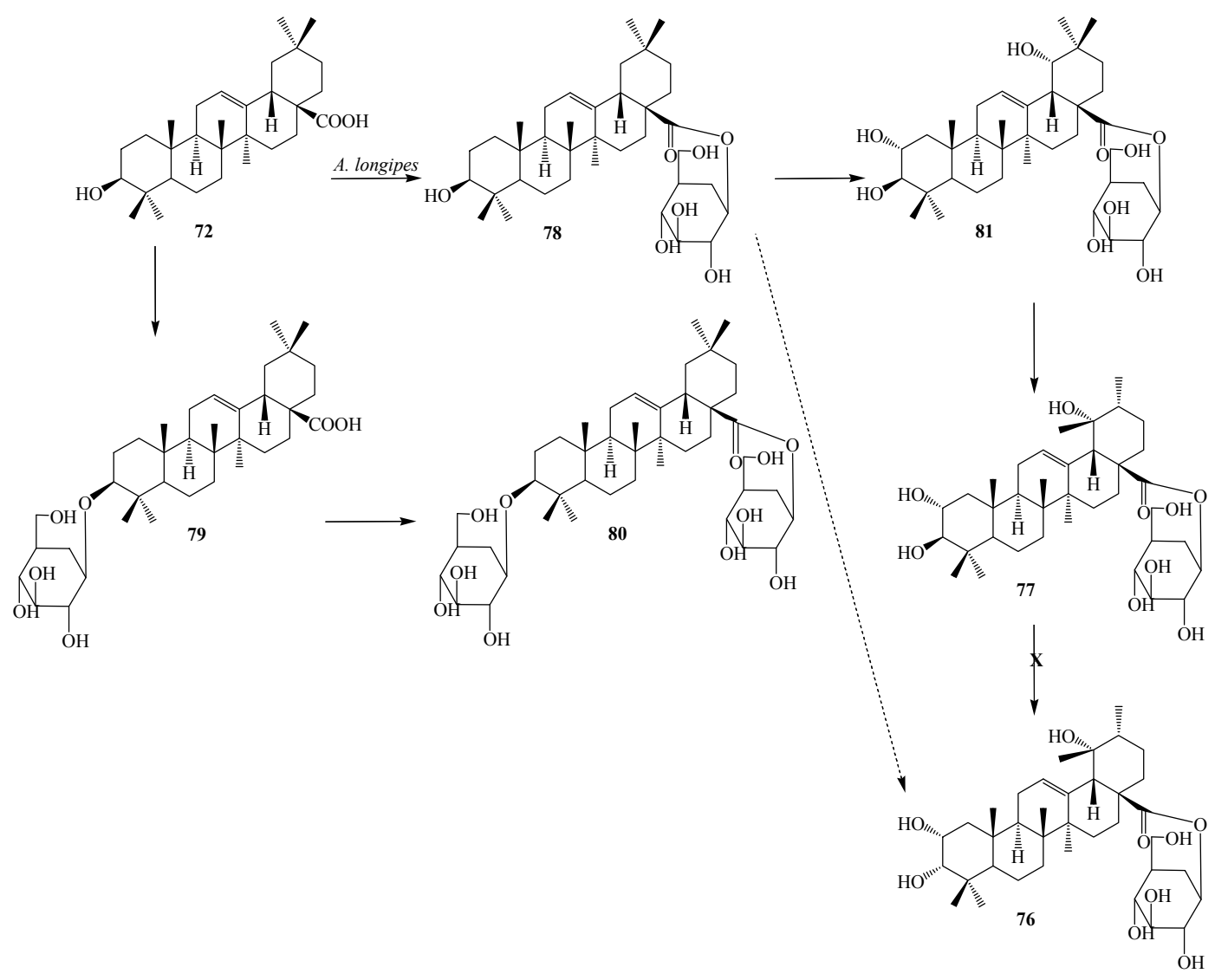

Figure 22. Microbial transformation of oleanolic acid (72) by Penicillium adametzi.

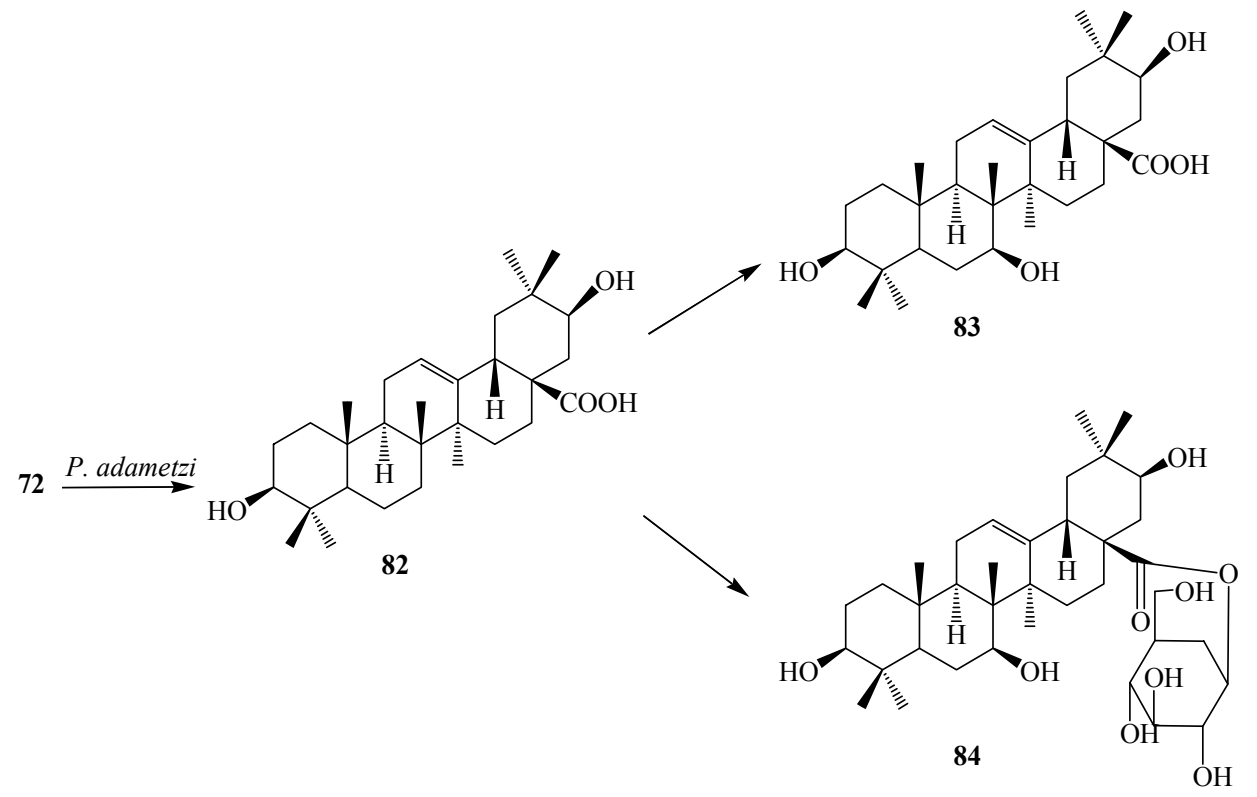

Chapel et al. reported the microbial transformation of $\mathbf{7 2}$ with the filamentous fungus, Mucor rouxii. Fermentation process yielded four regioselective derivatives, 83, 7 $\beta$-hydroxy-3-oxo-olean-12-en-28oic acid (85), $21 \beta$-hydroxy-3-oxo-olean-12-en-28-oic acid (86) and 7 $\beta, 21 \beta$-dihydroxy-3-oxo-olean-12en-28-oic acid (87) (Figure 23). Compound $\mathbf{8 6}$ displayed the potent activity against Porphyromonas gingivalis [35]. 
Martinez et al. investigated biotransformation of $\mathbf{7 2}$ with Rhizomucor miehei CECT 2749 and reported the production of a mixture of polar metabolites, 3ß,30-dihydroxyolean-12-en-28-oic acid (88),

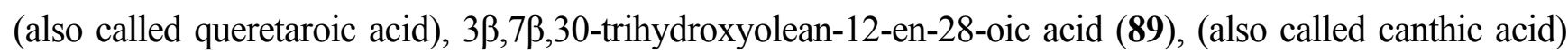

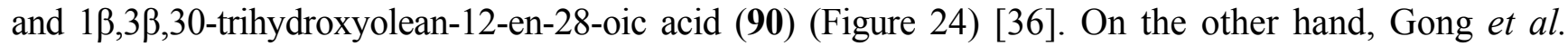
reported metabolism of $\mathbf{7 2}$ with Trichothecium roseum and showed the production of two hydroxylated metabolites, 15 $\alpha$-hydroxy-3-oxo-olean-12-en-28-oic acid (91) and 7 $\beta, 15 \alpha$-dihydroxy-3-oxo-olean-12en-28-oic acid (92) (Figure 25) [37].

Figure 23. Microbial transformation of oleanolic acid (72) by Mucor rouxii.

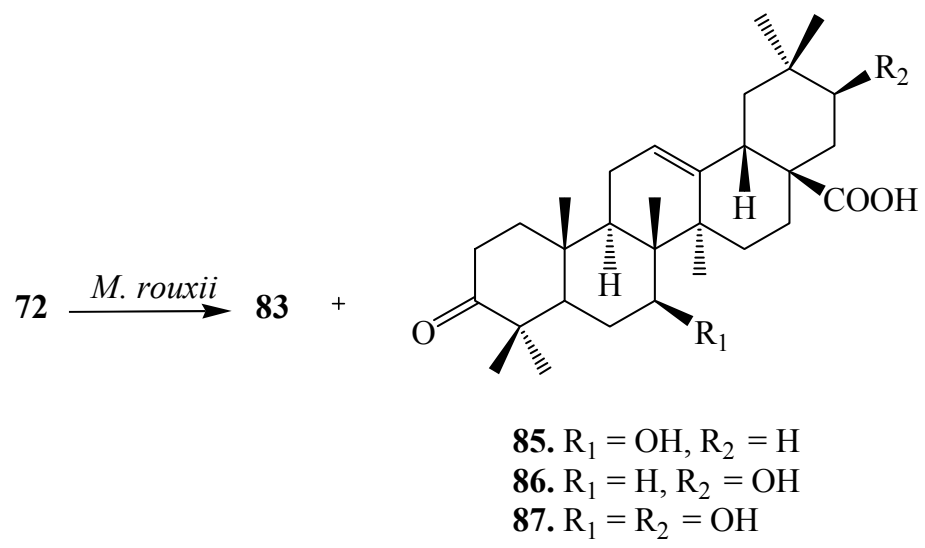

Figure 24. Metabolites isolated from the incubation of oleanolic acid (72) with Rhizomucor miehei.

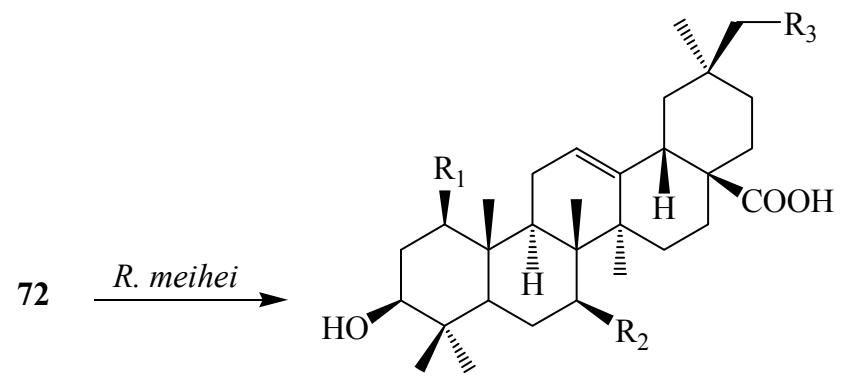

$$
\begin{aligned}
& \text { 88. } R_{1}=R_{2}=H, R_{3}=O H \\
& \text { 89. } R_{1}=H, R_{2}=R_{3}=O H \\
& \text { 90. } R_{1}=R_{3}=O H, R_{2}=H
\end{aligned}
$$

Figure 25. Metabolites isolated from the incubation of oleanolic acid (72) with Trichothecium roseum.

72

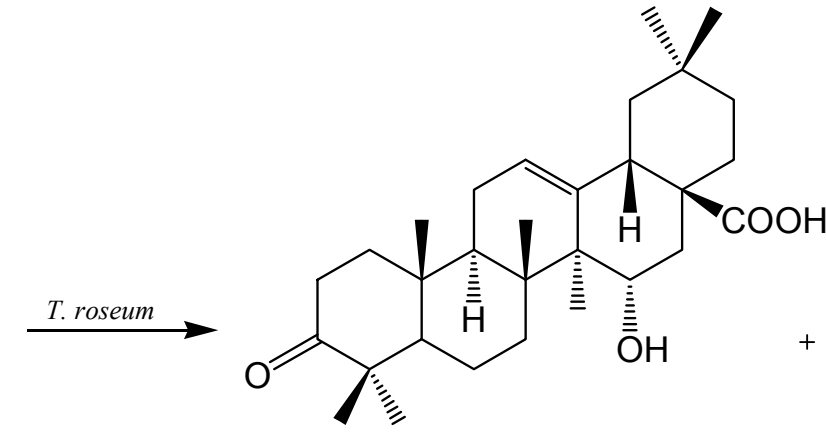

91

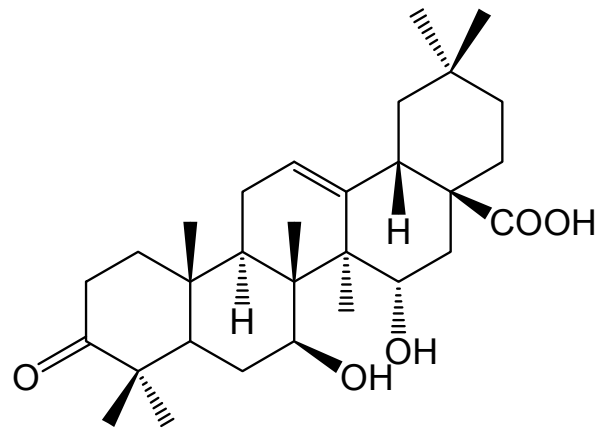

92 
Microbial transformation of $\mathbf{7 2}$ and three synthetic olean-type pentacyclic triterpenes, 3-oxo oleanolic acid (93), 3-acetyl oleanolic acid (94) and esculentoside A (95) with Streptomyces griseus ATCC 13273 and Aspergillus ochraceus CICC 40330 was reported by Zhu et al. (see Figures 26-30). These highly efficient and regioselective methyl oxidation and glycosylation provided an alternative approach to expand the structural diversity of OPTs [38]. The two interesting reactions observed during fermentation of four synthetic pentacyclic triterpenoids (3-4) with S. griseus ATCC 13273 and A. ochraceus CICC 40330, are the the regio-selective oxidation of the methyl group on C-4 and C-20 and the formation of glycosyl ester of C-28 carboxyl group. Fermentation of $\mathbf{7 2}$ with S. griseus ATCC 13273 yielded two more polar metabolites, serratagenic acid (96) and 33,24-dihydroxy-olean-12-en-28,29-dioic acid (97). Incubation of 72 with $A$. ochraceus CICC 40330 afforded another polar metabolite, 78 (Figure 26) [38].

Incubation of 3-oxo oleanolic acid (93) with S. griseus ATCC 13273 produced two polar metabolites, 3-oxo-olean-12-en-28,29-dioic acid (98) and 24-hydroxy-3-oxo-olean-12-en-28,29-dioic acid (99). On the other hand, incubation of 93 with A. ochraceus CICC 40330 afforded a different polar metabolite 28-O- $\beta$-D-glucopyranosyl 3-oxo-olean-12-en-28-oate (100) (Figure 27). The subsequent metabolism of $\mathbf{9 4}$ has also been reported. The bacterium $S$. griseus transformed 94 to two polar regioselective products, 96 and 97 (Figure 28). Compound 78, 28-O- $\beta$-D-glucopyranosyl,3 $\beta$ hydroxy-olean-12-en-28-oate was isolated from the culture of A. ochraceus CICC 40330 (Figure 28). Another substrate, esculentoside A (95) when incubated with $S$. griseus ATCC 13273 for 5 days yieled two less polar products, esculentoside B (104) and phytolaccagenin (105). Guo et al. investigated the regioselective bioconversion of $\mathbf{9 3}$ using the fungus Absidia glauca and reported the production of three novel hydroxylated metabolites, $1 \beta$-hydroxy-3-oxo-olean-11-en-28,13-lactone (101), $1 \beta, 11 \alpha$ dihydroxy-3-oxo-olean-12-en-28-oic acid (102), and 1 $\beta, 11 \alpha, 21 \beta$-trihydroxy-3-oxo-olean-12-en-28-oic acid (103) (Figure 29) [39].

Figure 26. Microbial transformation of oleanolic acid (72) by Streptomyces griseus ATCC 13273 and Aspergillus ochraceus CICC 40330.

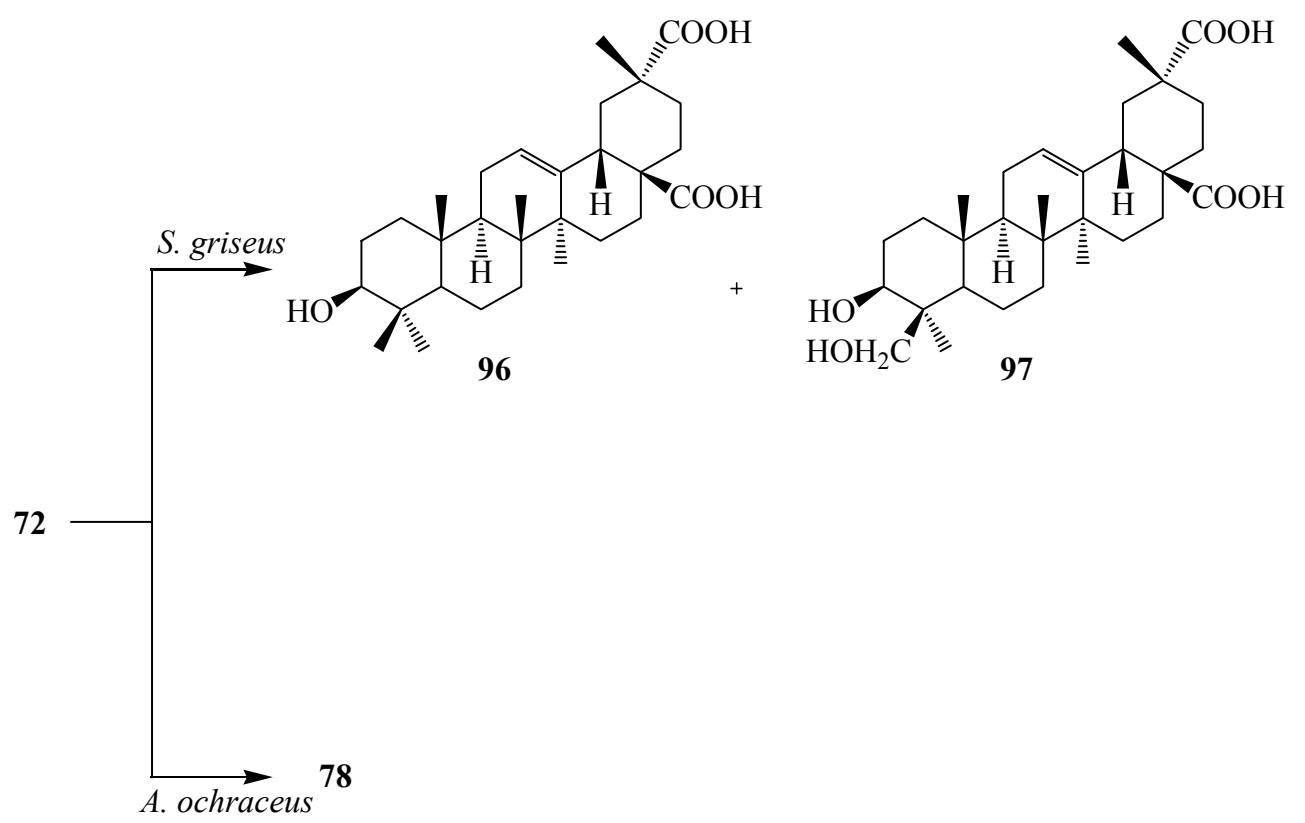


Figure 27. Microbial transformation of 3-oxo oleanolic acid (93) by Streptomyces griseus ATCC 13273 and Aspergillus ochraceus CICC 40330.

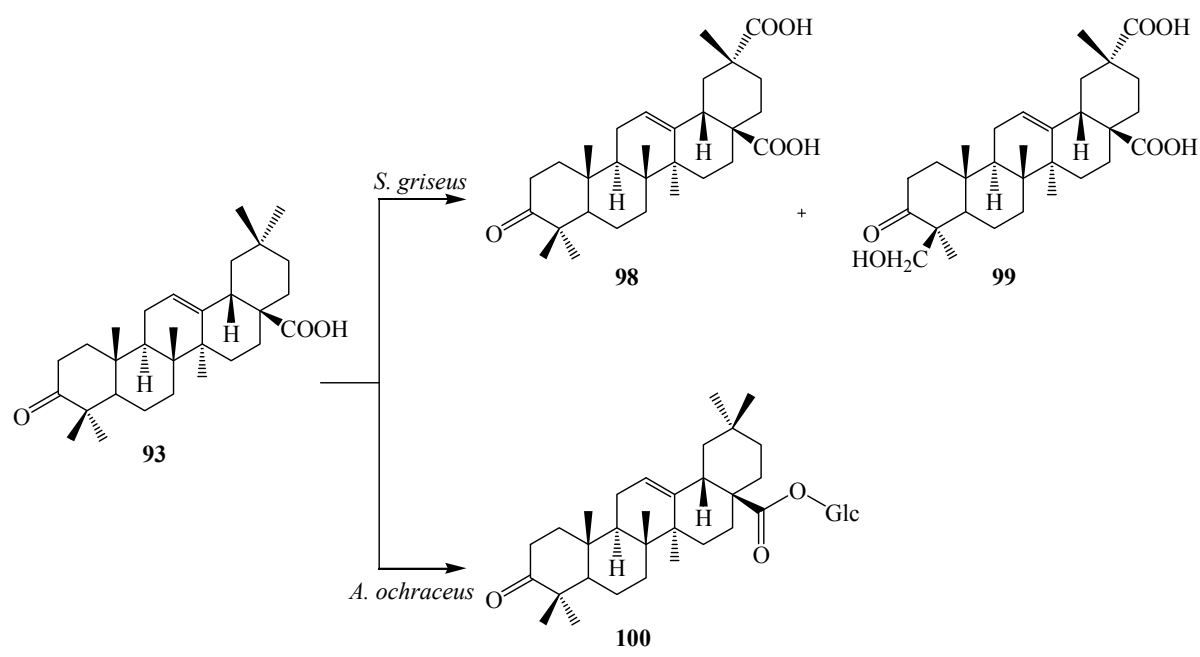

Figure 28. Microbial transformation of 3-acetyl oleanolic acid (94) by Streptomyces griseus ATCC 13273 and Aspergillus ochraceus CICC 40330.

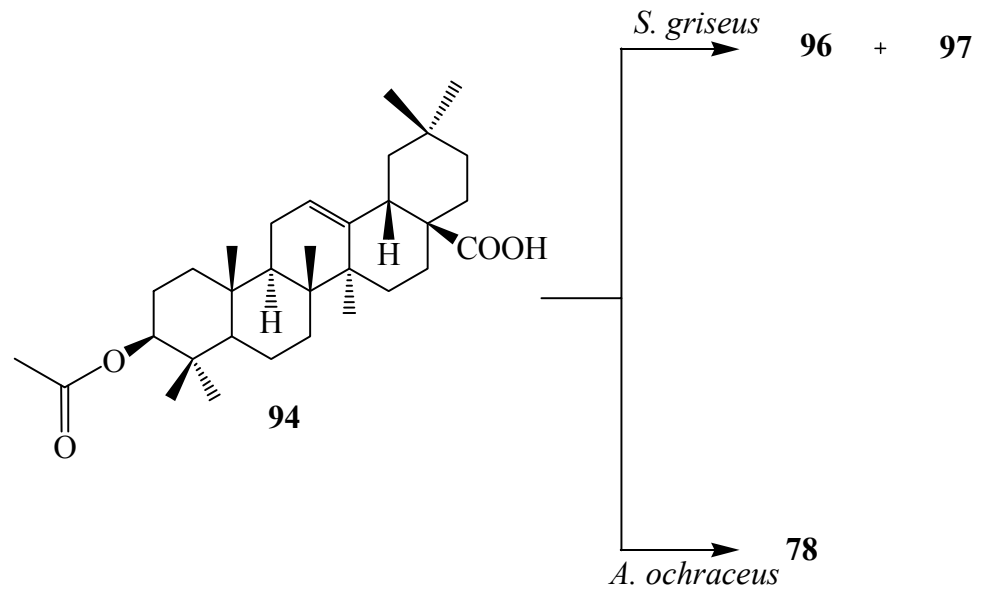

Figure 29. Microbial transformation of 3-oxo oleanolic acid (93) by growing cultures of the fungus Absidia glauca.

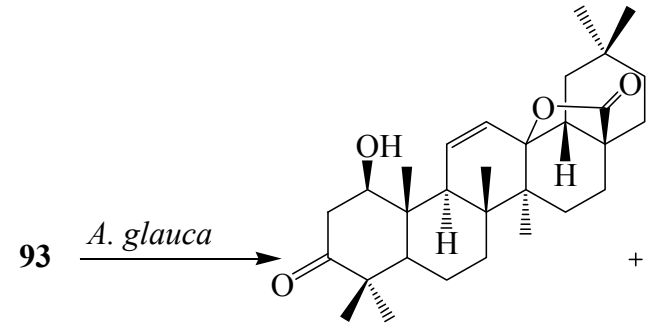

101

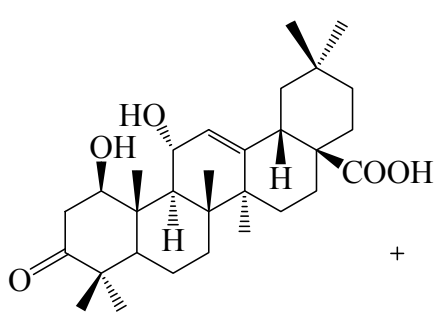

102

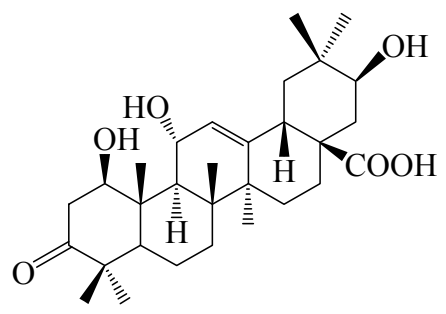

103

However, the incubation of 95 with A. ochraceus CICC 40330 afforded only metabolite $\mathbf{1 0 4 .}$

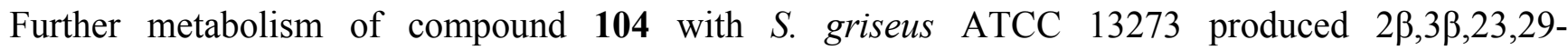
tetrahydroxy-olean-12-ene-28,30-dioic acid 30-methyl ester (106) and incubation of $\mathbf{1 0 5}$ with A. ochraceus CICC 40330 resulted 28-O- $\beta$-D-glucopyranosyl phytolaccagenin (107) (Figure 30) [38]. 
Figure 30. Microbial transformation of 3-oxo esculentoside A (95) by Streptomyces griseus ATCC 13273 and Aspergillus ochraceus CICC 40330.

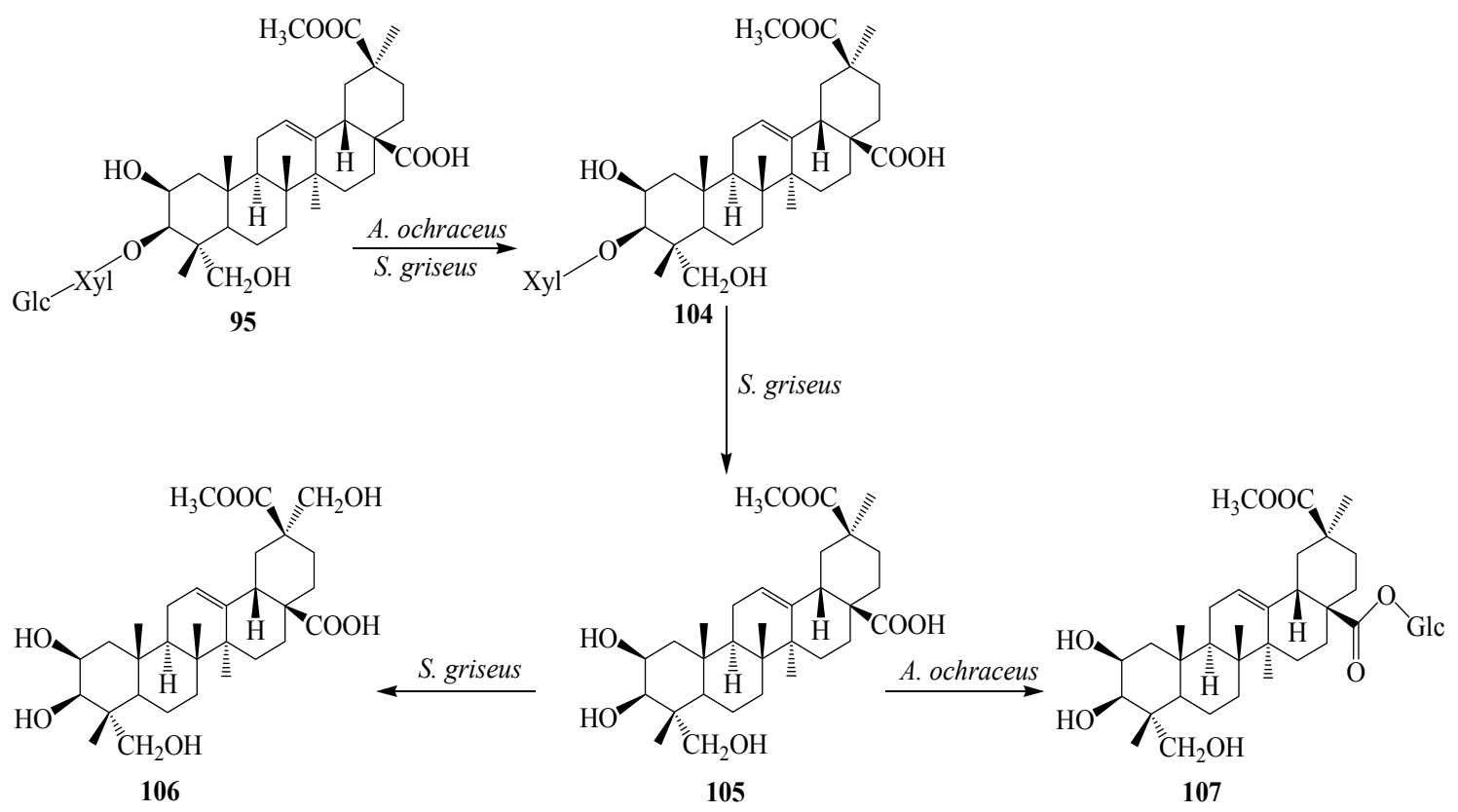

The pentacyclic triterpene maslinic acid $(2 \alpha, 3 \beta$-dihydroxyolean-12-en-28-oic acid, 73$)$ is a natural pentacyclic triterpenoid compounds which is present in abundant amount in the surface wax on the fruits and leaves of Olea europaea. It is also a byproduct of the solid waste obtained from olive oil production. This compound is also found in Agastache rugosa, Lagerstroemia speciosa, and Geum japonicum. Maslinic acid (73) has anti-HIV, anticancer, anti-diabetic, antioxidant and antiatherogenic activities. Martinez et al. investigated the metabolism of maslinic acid (73) with Rhizomucor miehei and reported the production of an olean-11-en-28,13 $\beta$-olide derivative, $2 \alpha, 3 \beta$-dihydroxyolean-11-en28,13 $\beta$-olide (108) and a hydroxylated product at C-30 position; $2 \alpha, 3 \beta, 30$-trihydroxyolean-12-en-28oic acid (109). These biotransformation reactions are shown in Figure 31 [36].

Feng et al. investigated the bioconversion of 73 by C. blakesleeana CGMCC 3.910 and demonstrated the formation of four derivatives, $2 \alpha, 3 \beta, 7 \beta$-trihydroxyolean-12-en-28-oic acid (110),

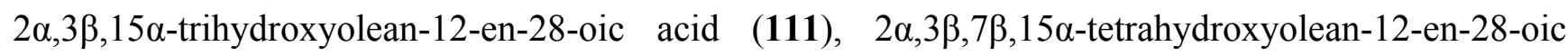
acid (112) and $2 \alpha, 3 \beta, 7 \beta, 13 \beta$-tetrahydroxyolean-11-en-28-oic acid (113) (Figure 32) [40].

Figure 31. Metabolites isolated from the incubation of maslinic acid (73) with Rhizomucor miehei.

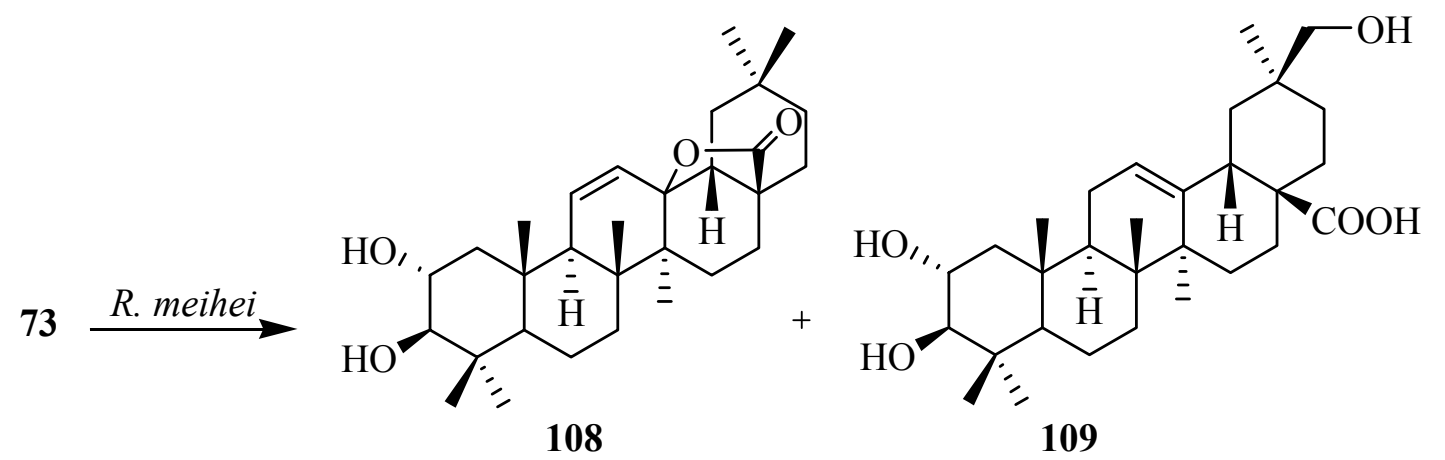


Figure 32. Microbial transformation of maslinic acid (73) by Cunninghamella blakesleeana.
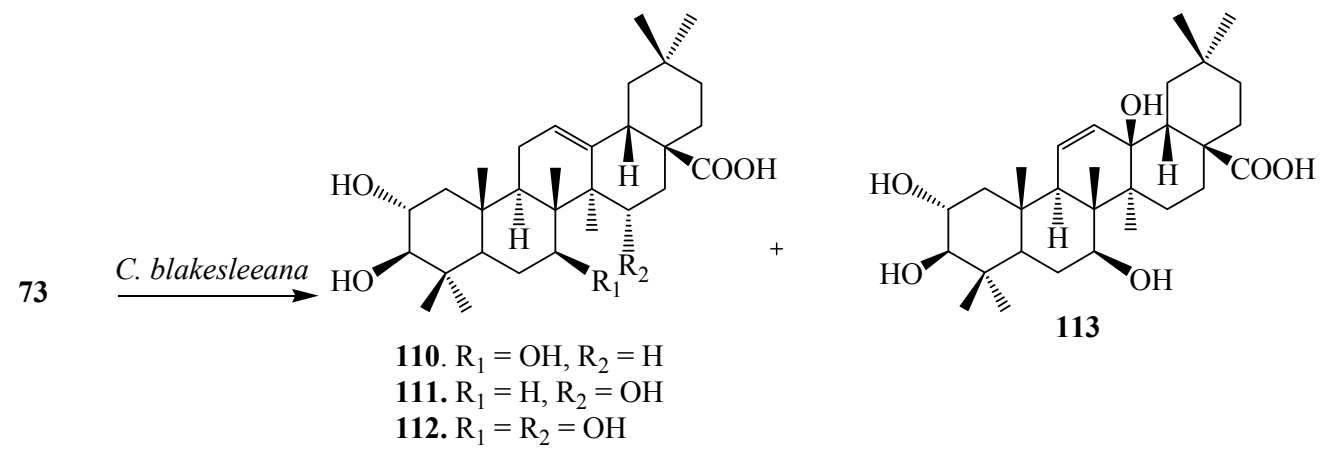

111. $\mathrm{R}_{1}=\mathrm{H}, \mathrm{R}_{2}=\mathrm{OH}$

112. $R_{1}=R_{2}=O H$

Ursolic acid (3ß-hydroxy-urs-12-en-28-oic acid, UA, 114), a pentacyclic triterpene acid, exists abundantly in many medicinal plants including sage, rosemary, thyme and lavender $[1,41,42]$. It displays a remarkable spectrum of biological activities, such as anti-inflammatory activity, anti-allergic activity, antibacterial activity, anti-mutagenicity, hepatoprotective activity, rantimalarial and anti-tumor activity. In addition, UA is also used to induce apoptosis in human liver cancer cell lines, to enhance the cellular immune system and pancreatic $\beta$-cell function and to inhibit invasion $[1,41]$.

Biotransformation of $\mathbf{1 1 4}$ by the filamentous fungus Syncephalastrum racemosum (Cohn) Schroter AS 3.264 was reported by Huang et al. They reported the regioselective hydroxylation, carbonylation,

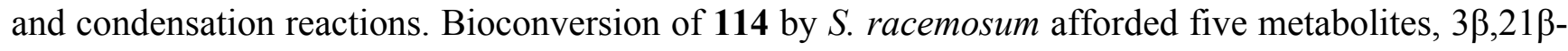

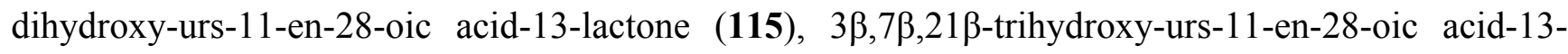

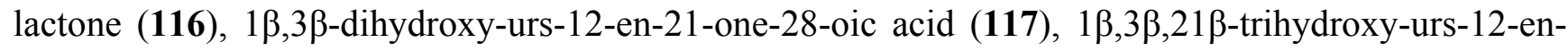

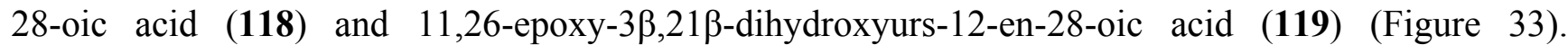
Compound 117 showed moderate PTP1B inhibitory activity [41].

$\mathrm{Fu}$ et al. conducted microbial transformation of $\mathbf{1 1 4}$ with filamentous fungus Syncephalastrum racemosum CGMCC 3.2500 (see Figure 34) and showed the formation of five polar metabolites 115, 116, 117, 118 and 3 $\beta, 7 \beta, 21 \beta$-trihydroxy-urs-12-en-28-oic acid (120). Metabolites 118 and 120 exhibited anti-HCV activity [42].

Figure 33. Fermentation of ursolic acid (114) with Syncephalastrum racemosum (Cohn) Schroter AS 3.264.

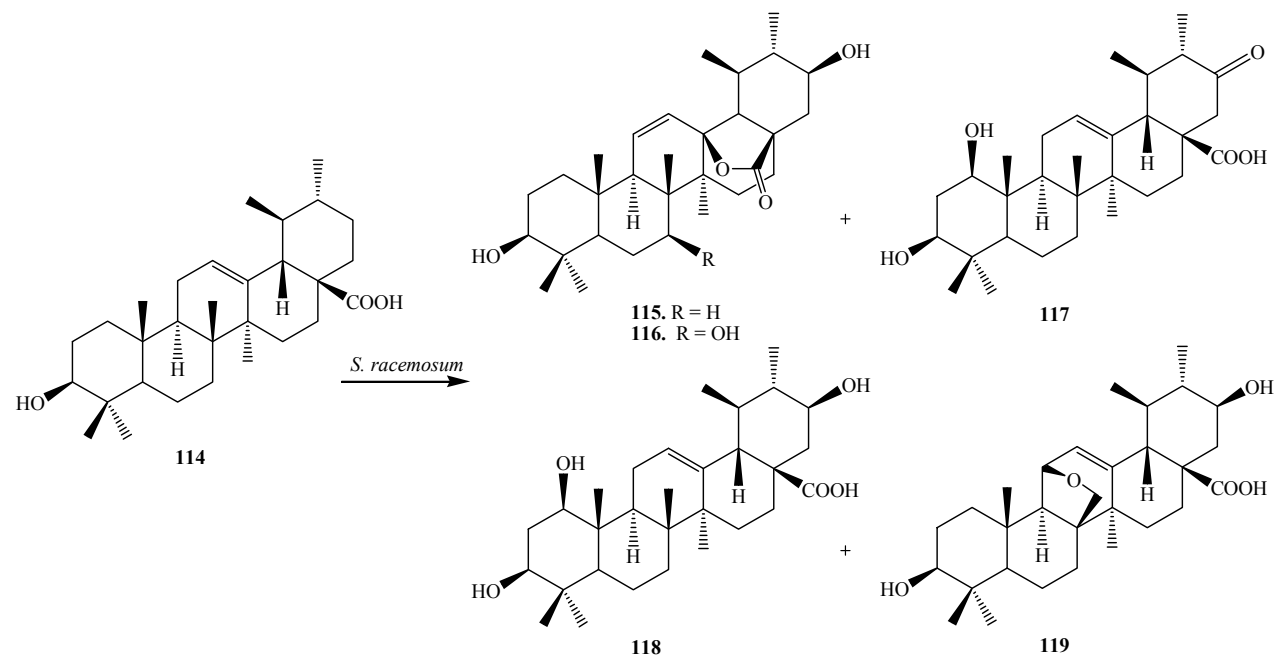


Figure 34. Biotransformation of ursolic acid (114) with Syncephalastrum racemosum CGMCC 3.2500.

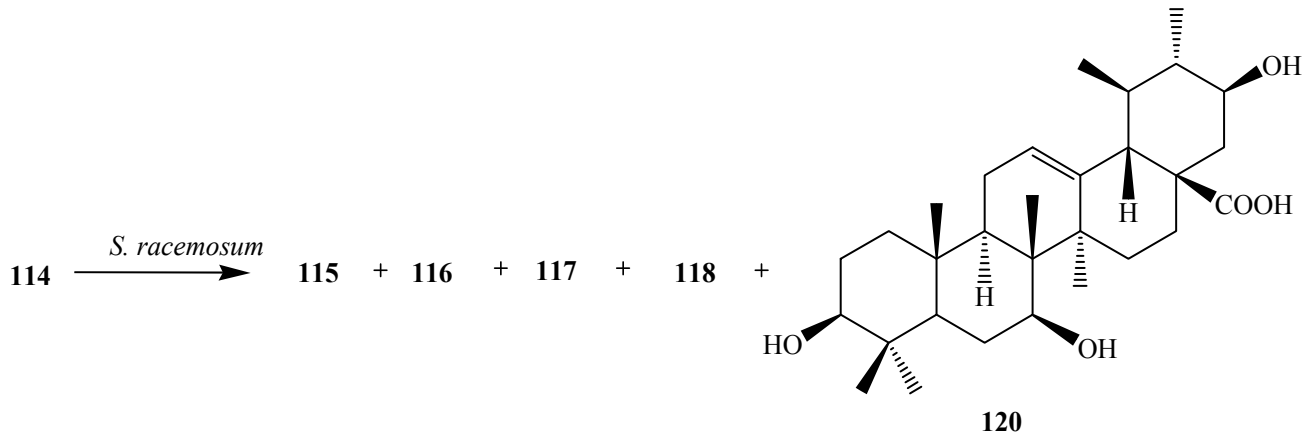

Endophytic microorganisms are bacterial or fungal organisms which colonize in the healthy plant tissue inter-and/or intracellularly without causing any apparent symptoms of disease. Endophytic microorganisms can produce bioactive natural substances, such as paclitaxel, podophyllotoxin etc. [43]. Endophytic fungi extensively metabolized 2-hydroxy-1,4-benzoxazin-3(2H)-one (HBOA) and biotransformed it to less toxic metabolites probable by their oxidase and reductases. Thus, endophytes attracted more and more attention not only for producing many novel substances but also to biotransform the natural products. Endophytic fungus, Umbelopsis isabellina, isolated from medicinal plant Huperzia serrata, was utilized to transform 114, into three regioselective products, $3 \beta$-hydroxy-

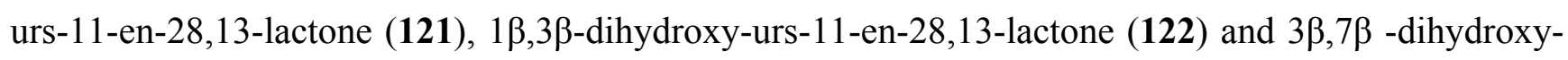
urs-11-en-28, 13-lactone (123) (Figure 35) [44].

Figure 35. Biotransformation of $\mathbf{1 1 4}$ by an endophytic fungus Umbelopsis isabellina.

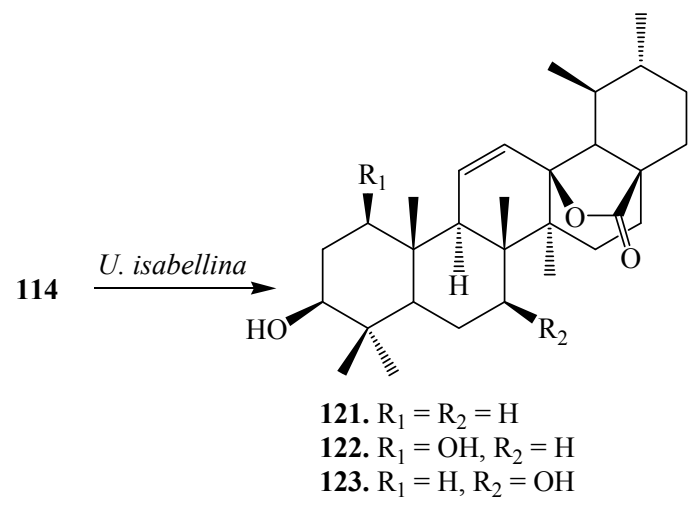

Microbial metabolism of triterpenoid 114 by various Nocardia sp. NRRL 5646, Nocardia sp. 44822 and Nocardia sp. 44000 was investigated by D. Leipold et al. Micobial conversion of 114 resulted methyl ester (124), ursonic acid (125), ursonic acid methyl ester (126), 3-oxoursa-1,12-dien-28-oic acid (127) and 3-oxoursa-1,12-dien-28-oic acid methyl ester (128). Nocardia sp. 45077 synthesized ursonic acid (125) and 3-oxoursa-1,12-dien-28-oic acid (127), while Nocardia sp. 46002 produced only ursonic acid (125). Nocardia sp. 43069 did not cause any biotransformation of this compound [45]. Microbial metabolism of 114 by Aspergillus flavus (ATCC 9170) was studied by Ibrahim et al., who showed the formation of 3-oxo ursolic acid derivative, ursonic acid (125). These transformation reactions are shown in Figure 36 [46]. 
Figure 36. Fermentation of ursolic acid (114) with Nocardia sp. and Aspergillus flavus.

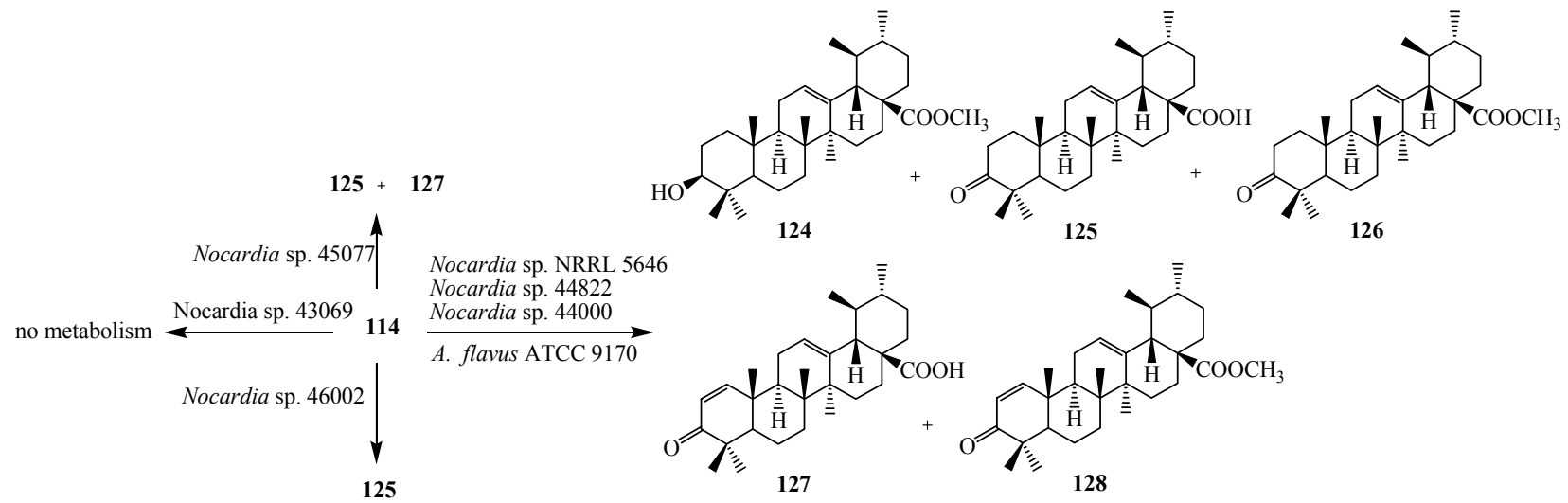

Microbial modification of $\mathbf{1 1 4}$ by an endophytic fungus Pestalotiopsis microspora was carried out by $\mathrm{S}$. Fu et al. Incubation of $\mathbf{1 1 4}$ with $P$. microspora afforded four metabolites: 3-oxo-15 $\alpha$,

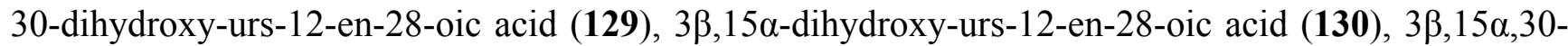
trihydroxy-urs-12-en-28-oic acid (131) and 3,4-seco-ursan-4,30-dihydroxy-12-en-3,28-dioic acid (132) (Figure 37) [47].

Lupane terpenoids are a group of pentacyclic triterpenoids that consist of compounds with antimalarial, vasorelaxant activities, and potent inhibitors against glycogen phosphorylase. Filamentous fungi, Aspergillus ochraceus metabolized lupeol (133) to two derivatives 134 and 135 (Figure 38). Fermentation of $\mathbf{1 3 3}$ with Mucor rouxii for 240 h yielded two other polar products: 136 and 137 [48].

Figure 37. Microbial metabolism of ursolic acid (114) with endophytic fungus Pestalotiopsis microspora.

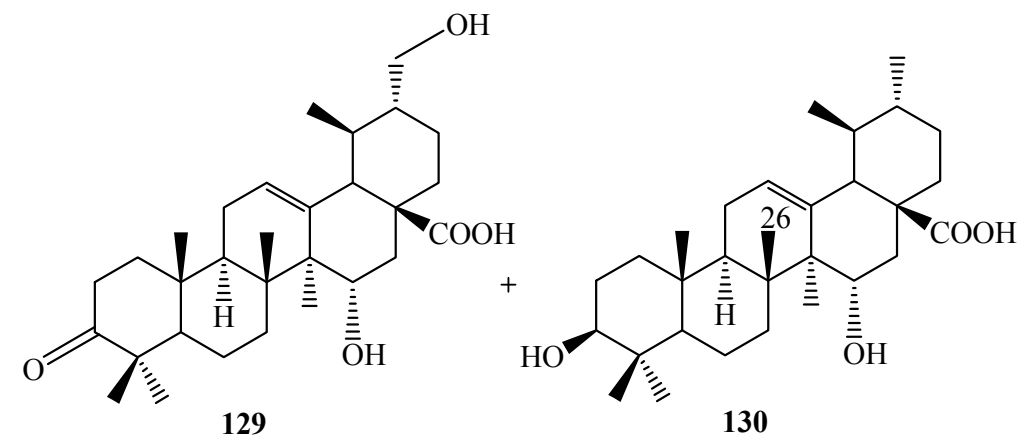

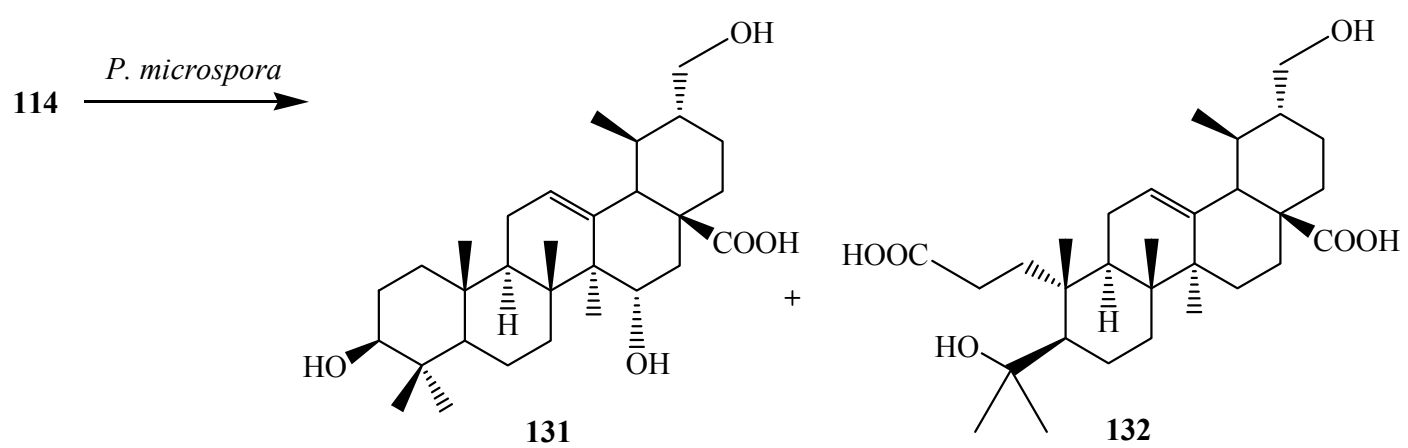


Figure 38. Biotransformation of lupeol (133), produced by Aspergillus ochraceus and Mucor rouxii.

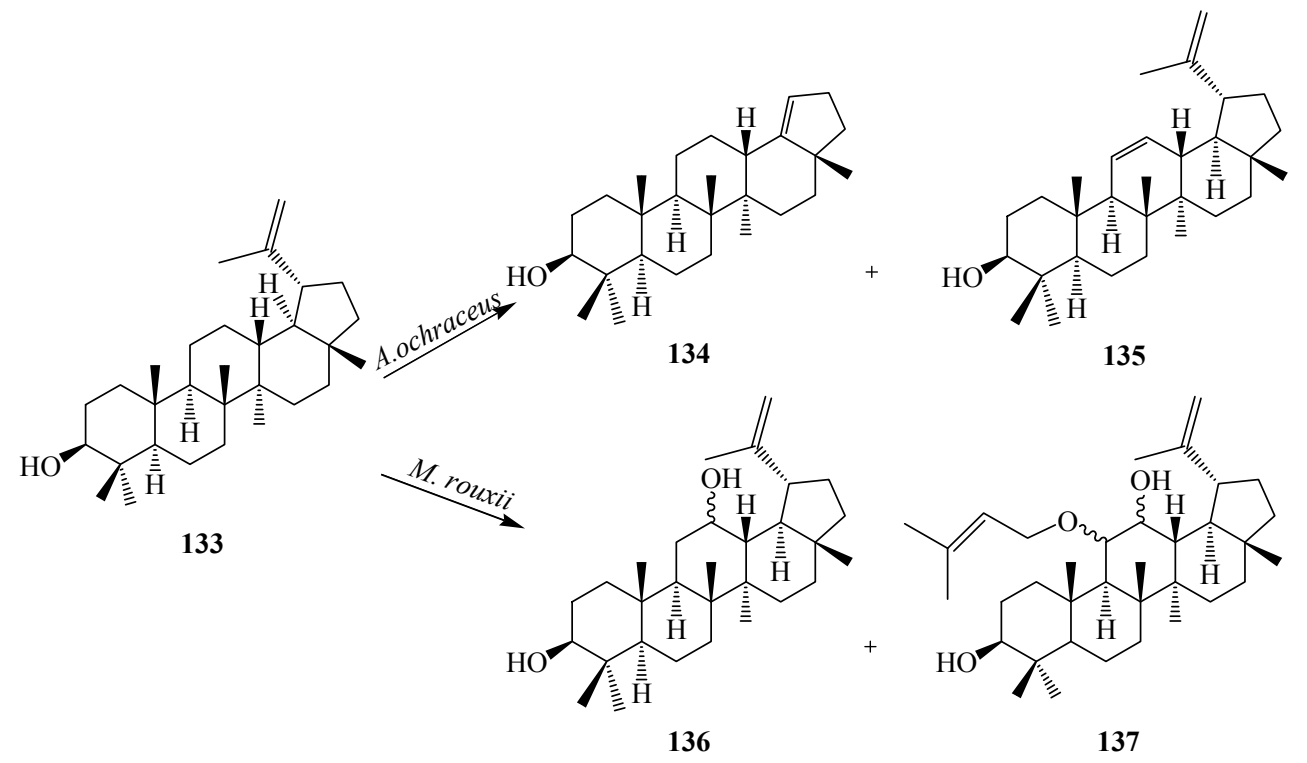

18ß-Glycyrrhetinic acid (138) is the active form of glycyrrhizin which is the major pentacyclic triterpene found in licorice (Glycyrrhiza glabra L.). It is one of the principal constituents of traditional Chinese herbal medicine, the rhizome of Glycyrrhiza uralensis (called Gancao). Glycyrrhetinic acid (138) has been shown to possess several pharmacological activities, such as antiulcerative, anti-inflammatory, immunomodulating, antitumour activities, antiviral activity, interferon-inducibility, antihepatitis effects and anticancer activity. Several hydroxy derivatives of $\mathbf{1 3 8}$ enhanced anti-inflammatory, antioxidant activities, anti-proliferative and apoptotic activities. Several derivatives of $\mathbf{1 3 8}$ have already been used as pharmaceutical drugs [49-51]. Many microorganisms catalyze 138 to its derivatives with structural diversity. Qin et al. investigated metabolism of $\mathbf{1 3 8}$ with a fungus, Cunninghamella blakesleeana AS 3.970 and reported the production of 3-oxo-7 $\beta$-hydroxyglycyrrhetinic acid (139) and 7 $\beta$-hydroxyglycyrrhetinic acid (140) (Figure 39). Both metabolites showed activities against drug-resistant Enterococcus faecalis [49].

Xin et al. investigated the microbial transformation of $\mathbf{1 3 8}$ by Mucor polymorphosporus. Incubating 138 with $M$. polymorphosporus (Figure 40) for 10 days yielded five polar products: 6 $\beta, 7 \beta-$ dihydroxyglycyrrhentic acid (141), 27-hydroxyglycyrrhentic acid (142), 24-hydroxyglycyrrhentic acid (143), 3-oxo-7 $\beta$-hydroxyglycyrrhentic acid (144) and 7 $\alpha$-hydroxyglycyrrhentic acid (145) [50].

Figure 39. Biotransformation of glycyrrhetinic Acid (138) by Cunninghamella blakesleeana.

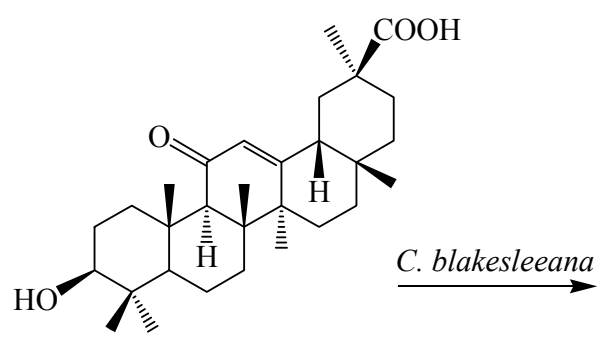

138

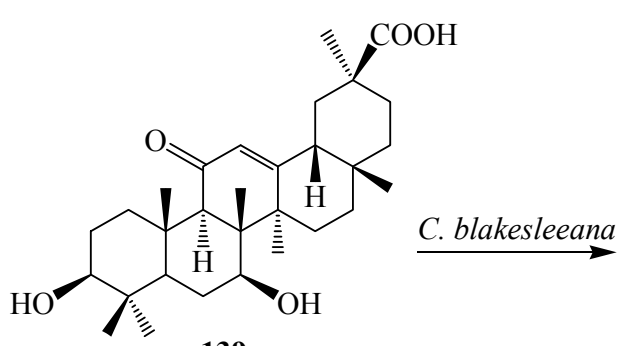

139

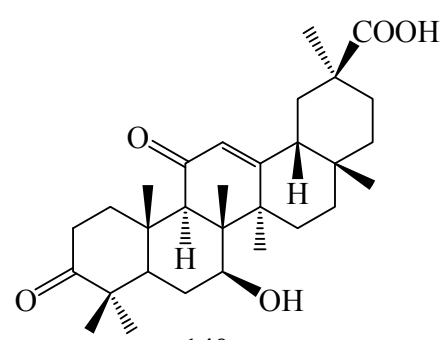

140 
Figure 40. Biotransformation of $\mathbf{1 3 8}$ by Mucor polymorphosporus.

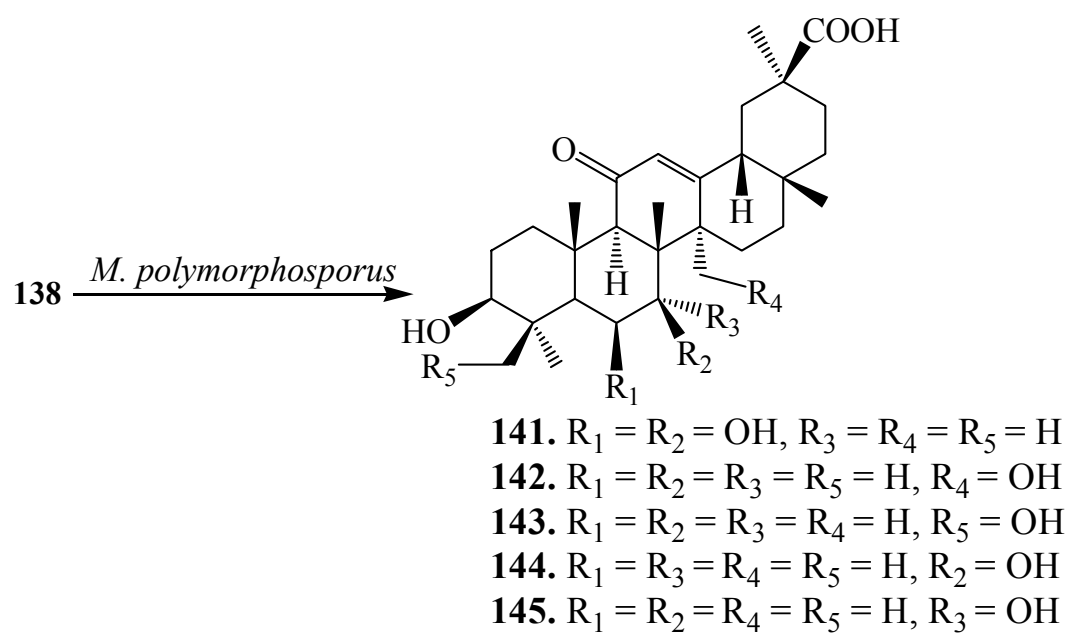

Bioconversion of 138 using Absidia pseudocylindrospora ATCC 24169, Gliocladium viride ATCC 10097 and Cunninghamella echinulata ATCC 8688a was carried out by G.T. Maatooq et al. Fermentation of $\mathbf{1 3 8}$ afforded seven polar derivatives: $7 \beta, 15 \alpha$-dihydroxy-18 $\beta$-glycyrrhetinic acid

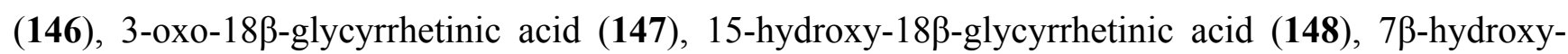

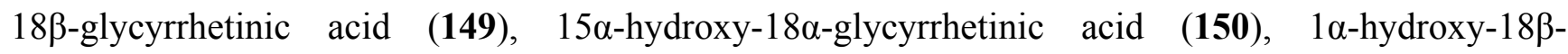

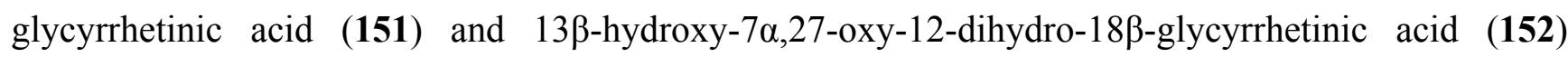
(Figure 41). Compound 138, 146 and 150 enhanced the production of Nitric oxide (NO) in peritoneal rat macrophages treated with $\mathrm{CCl}_{4}[51]$.

Figure 41. Biotransformation of $\mathbf{1 3 8}$ by Absidia pseudocylindrospora ATCC 24169, Gliocladium viride ATCC 10097 and Cunninghamella echinulata ATCC 8688a.

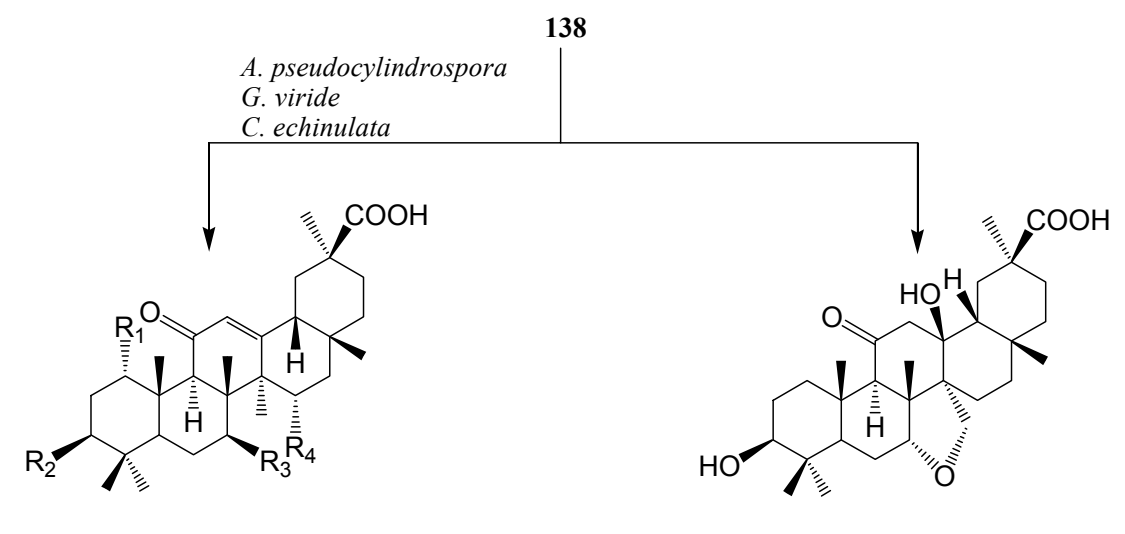

$\begin{array}{llll}\mathrm{R}_{1} & \mathrm{R}_{2} & \mathrm{R}_{3} & \mathrm{R}_{4} \\ \text { 146. } \mathrm{H} & \mathrm{OH} & \mathrm{OH} & \mathrm{OH} \\ \text { 147. } \mathrm{H} & \mathrm{O} & \mathrm{H} & \mathrm{H} \\ \text { 148. } \mathrm{H} & \mathrm{OH} & \mathrm{H} & \mathrm{OH} \\ \text { 149. } \mathrm{H} & \mathrm{OH} & \mathrm{OH} & \mathrm{H} \\ \text { 150. } \mathrm{H} & \mathrm{OH} & \mathrm{H} & \mathrm{OH} \\ \text { 151. } \mathrm{OH} & \mathrm{OH} & \mathrm{H} & \mathrm{H}\end{array}$

152

Betulin (lup-20(29)-ene-3 $\beta$, 28-diol, 153), also known as betulinic alcohol, is a pentacyclic triterpene alcohol with a lupane skeleton. The most widely reported source of $\mathbf{1 5 3}$ is the birch tree (Betula sp.). It is also called betulinol and is structurally similar to sitosterols. It has estrogenic 
properties. Betulinic acid (154), 3 -hydroxy-lup-20(29)-en-28-oic acid, an antimalarial triterpenoid present in many plant species such as birch tree (Betula sp.), Ziziphus sp., Syzygium sp., Diospyros sp. and Paeonia sp. [6]. It has attracted more and more attention due to its important physiological and pharmacological activities such as antitumor, anti-HIV, antiviral, anti-leukaemia, anti-inflammatory, antimicrobial, antihelmintic, anti-feedant activities, antimalarial and anticancer activities [6]. Betulinic acid (154) and its derivatives are also potential bioactive compounds present in nature [52,53]. Biotransformation of betulin (153) was carried out by Chen et al. with the filamentous fungi, Armillaria luteo-virens Sacc QH (ALVS), Aspergillus foetidus ZU-G1 (AF) and Aspergillus oryzae (AO), which resulted 154 in 6 days. Furthermore, Y. Feng et al. investigated biotransformation of 153 to $\mathbf{1 5 4}$ by Cunninghamella blakesleeana cells in 6 days of fermentation. These microbial reactions are described rin Figure 42 [52,53].

Figure 42. Microbial transformation of betulin (153) to betulinic acid (154) by Cunninghamella blakesleeana, Armillaria luteo-virens Sacc, Aspergillus foetidus and Aspergillus oryzae.

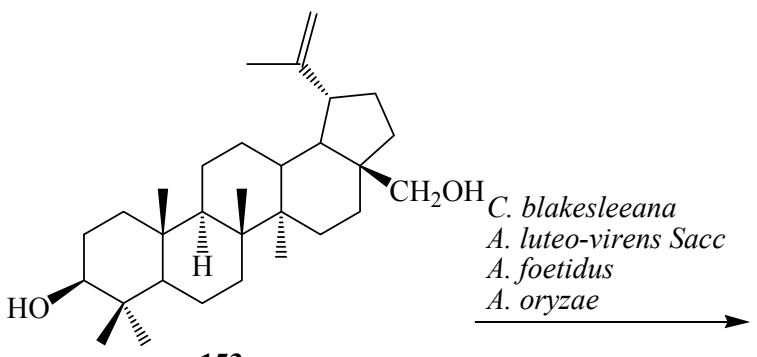

153

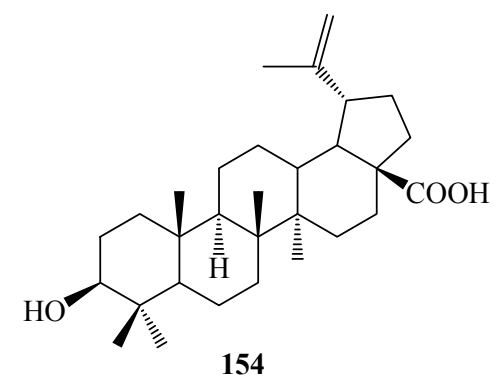

154

Grishko et al. reported regioselective oxidation of $\mathbf{1 5 3}$ into 3-oxo derivative, betulone (155) by the resting cells of the actinobacterium Rhodococcus rhodochrous IEGM 66 (Figure 43) [54]. Mao et al. reported the fermentation of betulin (153) by the yeast Rhodotorula mucilaginosa. This resulted in the production of compound 155. Compound $\mathbf{1 5 5}$ exhibited 2 times higher antioxidative activity than that of 153. Regioselective oxidation of 153 into 155 with growing microorganism cells of marine fungi Dothideomycete sp. HQ 316564 was reported by Li et al. [55,56].

Figure 43. Biotransformation of 153 to betulone (155) by Rhodococcus rhodochrous, Rhodotorula mucilaginosa and growing cultures of marine fungus Dothideomycete sp. HQ 316564 at $\mathrm{pH} 6$.

153

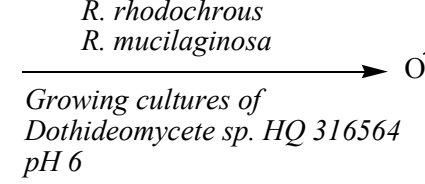


Preparation of betulinic acid derivatives through groups of fungi, such as Mycelia sterilia, Penicillium citreonigrum and Penicillium sp. was investigated by Baratto et al. M. sterilia converted $\mathbf{1 5 4}$ to an antimalarial agent [6], betulonic acid (156), Penicillium sp. biotransformed 154 to 156 and methyl 3-oxolup-20(29)-en-28-oate (157), and $P$. citreonigrum transformed $\mathbf{1 5 6}$ to methyl 3-hydroxylup-20(29)-en-28-oate (158). Biotranformation of 156 with carrot cell yielded 3-hydroxy(20R)-29-oxolupan-28-oic acid (159) in 14 days [57]. These biotransformations are shown in Figure 44.

Figure 44. Biotransformation of betulonic acid (154) with Mycelia sterilia, Penicillium sp. Penicillium citreonigrum and Daucus carota cells suspension.

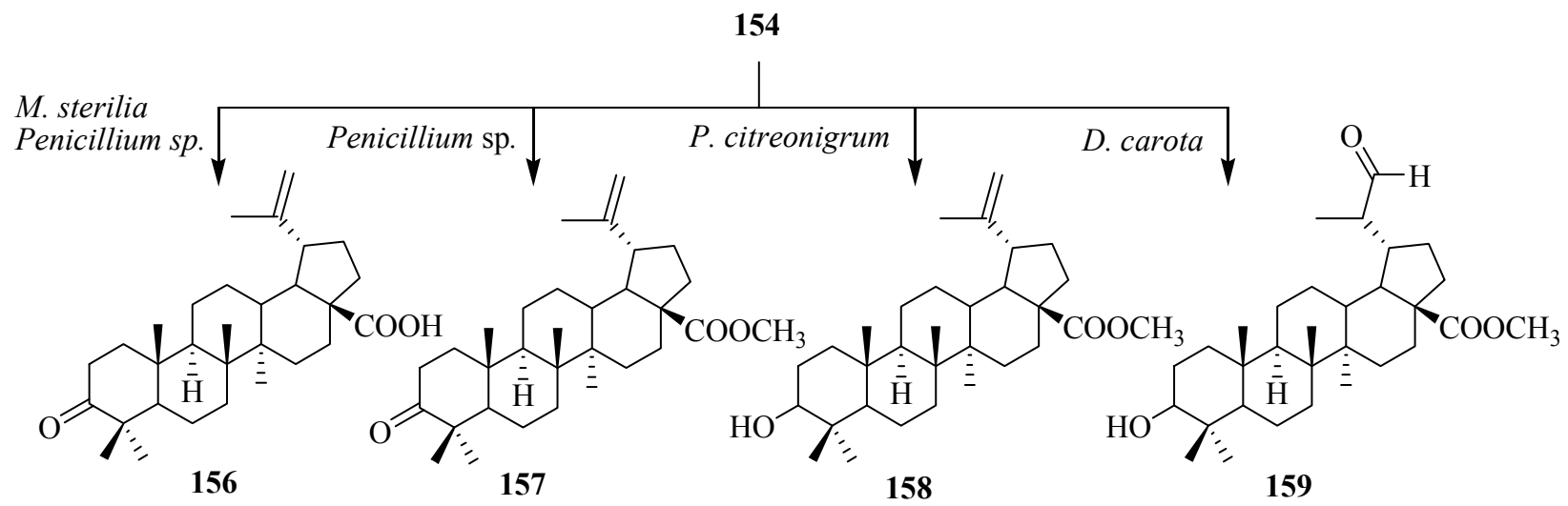

Nocardia sp. NRRL 5646 has been shown to produce a complex set of natural products to generate diverse structures [58]. It has been used extensively to catalyze numerous biotransformations, including carboxylic acid and aldehyde reduction, phenol methylation, and flavone hydroxylation. Microbial transformation of $\mathbf{1 5 6}$ by Nocardia sp. NRRL 5646 was investigated by Qian et al. Fermentation of $\mathbf{1 5 6}$ for 6 days yielded asymmetric $\alpha$-hydroxylation product, methyl $2 \alpha$-acetoxy-3-oxo-lup-20(29)-en-28-oate (160) and a methyl esterification of the C-28 carboxyl group, methyl 3-oxo-lup-20(29)-en-28-oate (161) (Figure 45) [59].

Figure 45. Microbial transformation of $\mathbf{1 5 6}$ by Nocardia sp. NRRL 5646.
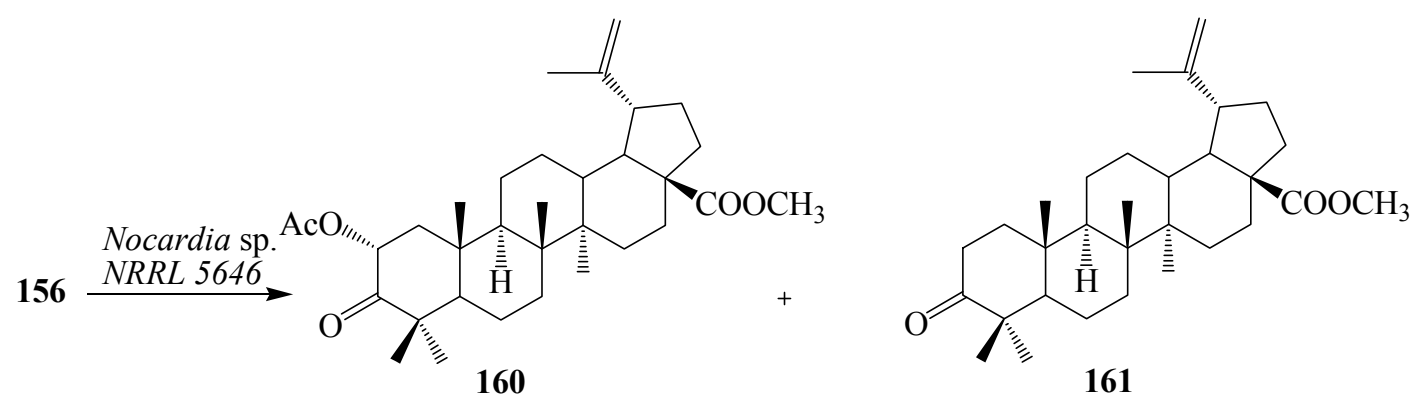

Phytolaccagenin (2 $\beta, 3 \beta, 23$-trihydroxy-olean-12-ene-28,30-dioic acid 30-methyl ester, 162), a major aglycone constituent found in rPhytolacca esculenta van Houtte. Phytolacca esculenta is widely distributed in East Asia and is used as a crude drug against edema, theumatism, bronchitis and tumors in China, Korea and Japan. The roots of P. esculenta are rich source of saponins and possess anti-inflammatory properties. They also induced immune interferons and tumor necrosis factor [60]. Compound 162 exhibits high activity against acute inflammation. Regiospecific hydroxylation on the 
C-29 methyl group of $\mathbf{1 6 2}$ by Streptomyces griseus ATCC 13273 was reported by Qian et al.

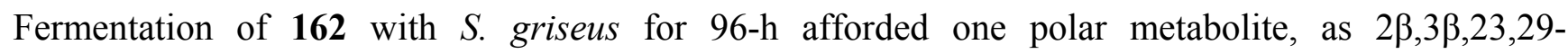
tetrahydroxy-olean-12-ene-28,30-dioic acid 30-methyl ester (163) as shown in Figure 46 [60].

Figure 46. Regio-specific microbial hydroxylation of phytolaccagenin (162) by Streptomyces griseus.

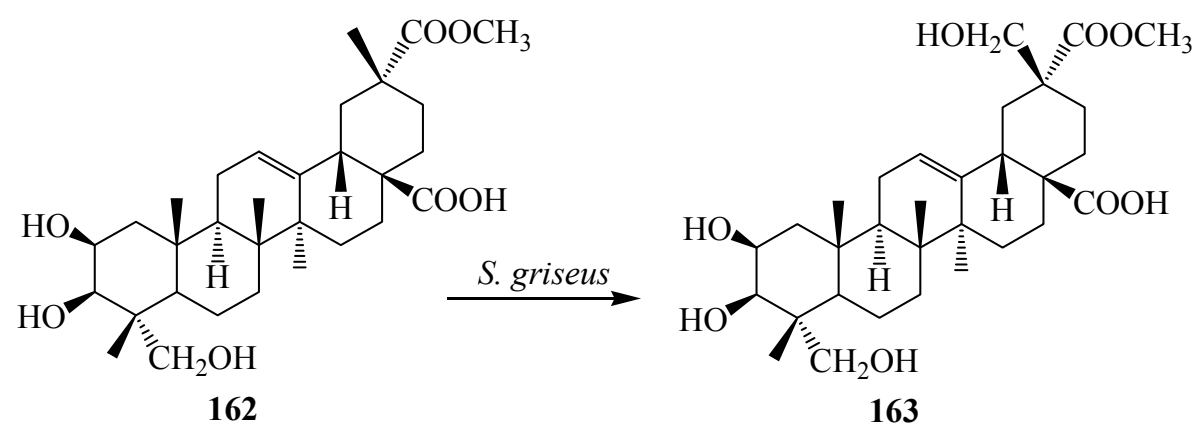

The gum resin of Boswellia serrata has been used for the treatment of inflammatory and arthritic diseases. Its major active constituents are ursane triterpenoids, boswellic acids (BAs), which include 11-keto- $\beta$-boswellic acid (KBA, 164) (Figure 47), acetyl-11-keto- $\beta$-boswellic acid (AKBA), $\beta$-boswellic acid (BA) and acetyl- $\beta$-boswellic acid (ABA). Among these, AKBA and KBA, possessing an 11-keto group, are the most bioactive compounds. They exhibited significant biological activities, including anti-inflammatory, anti-arthritis, anti-ulcerative colitis, anti-asthma, anticancer, and anti-hepatitis B properties [61]. Microbial rmetabolism of $\mathbf{1 6 4}$ by Cunninghamella blakesleeana AS 3.970 was studied by Wang et al. Fermentation of 164 by $C$. blakesleeana for 5 days yielded ten regioselective transformed products, which were characterized as 7 $\beta$-hydroxy-11-keto- $\beta$-boswellic acid (165); 7 $\beta, 15 \alpha$-dihydroxy-11-keto- $\beta$-boswellic acid (166); 7 $\beta, 16 \beta$-dihydroxy-11-keto- $\beta$-boswellic acid (167); 7 $\beta, 16 \alpha$-dihydroxy-11-keto- $\beta$-boswellic acid (168); 7 $\beta, 22 \beta$-dihydroxy-11-keto- $\beta$-boswellic

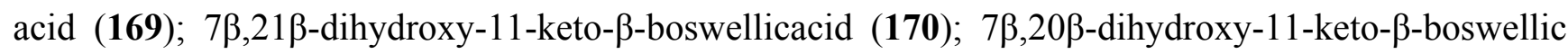
acid (171); 7 $\beta, 30$-dihydroxy-11-keto- $\beta$-boswellic acid (172); 3 $\alpha, 7 \beta$-dihydroxy-11-oxours-12-ene-24, 30-dioic acid (173) and 3 $\alpha, 7 \beta$-dihydroxy-30-(2-hydroxypropanoyloxy)-11-oxours-12-en-24-oic (174). These fungal transformation reactions are depicted in Figure 47. Compound $\mathbf{1 6 7}$ and 171 exhibit significant inhibitory effect on nitric oxide (NO) production in RAW 264.7 macrophage cells [55]. The location of the hydroxyl functionalities were deduced on the basis of the heteronuclear multiple bond connectivity (HMBC) interactions whereas orientations of $\mathrm{OH}$ groups were deduced on the basis of NOESY correlations [61].

Fermentation of $\mathbf{1 6 4}$ with Bacillus megaterium based on a recombinant cytochrome P450 system was reported by Bleif et al. Metabolism of $\mathbf{1 6 4}$ yielded regio- and stereoselective 15a-hydroxylation of substrate 164 (Figure 47). The structure was identified as 15 $\alpha$-hydroxy-KBA (175) by NMR spectroscopy [62].

Hepatitis $\mathrm{C}$ virus (HCV) infection is the leading cause of liver fibrosis and cirrhosis which eventually leads to liver cancer. Echinocystic acid (3 $\beta, 16 \alpha$-dihydroxy-olean-12-en-28-oic acid, 176) (Figure 48) is an oleanane-type triterpene, obtained from Echinocystis fabacea that exhibits sustantial inhibition of HCV. Echinocystic acid (176) and its saponins have been reported to have cytotoxic effects against different cell lines, including the J774.A1, HEK-293, WEHI-164 cell lines, the HepG2, 
HL-60 cells, the A375, Hela, and L929 cell lines in vitro. Echinocystic acid (176) and its saponins have many other bioactivities, including anti-HIV activities, antifungal activities, inhibitory activity toward pancreatic lipase, immunostimulatory effect, inhibition of mast cell degranulation, and the interleukin-18 inhibitory activities [63,64].

Figure 47. Biotransformation of 11-keto- $\beta$-boswellic acid (164) by Cunninghamella blakesleeana and Bacillus megaterium.

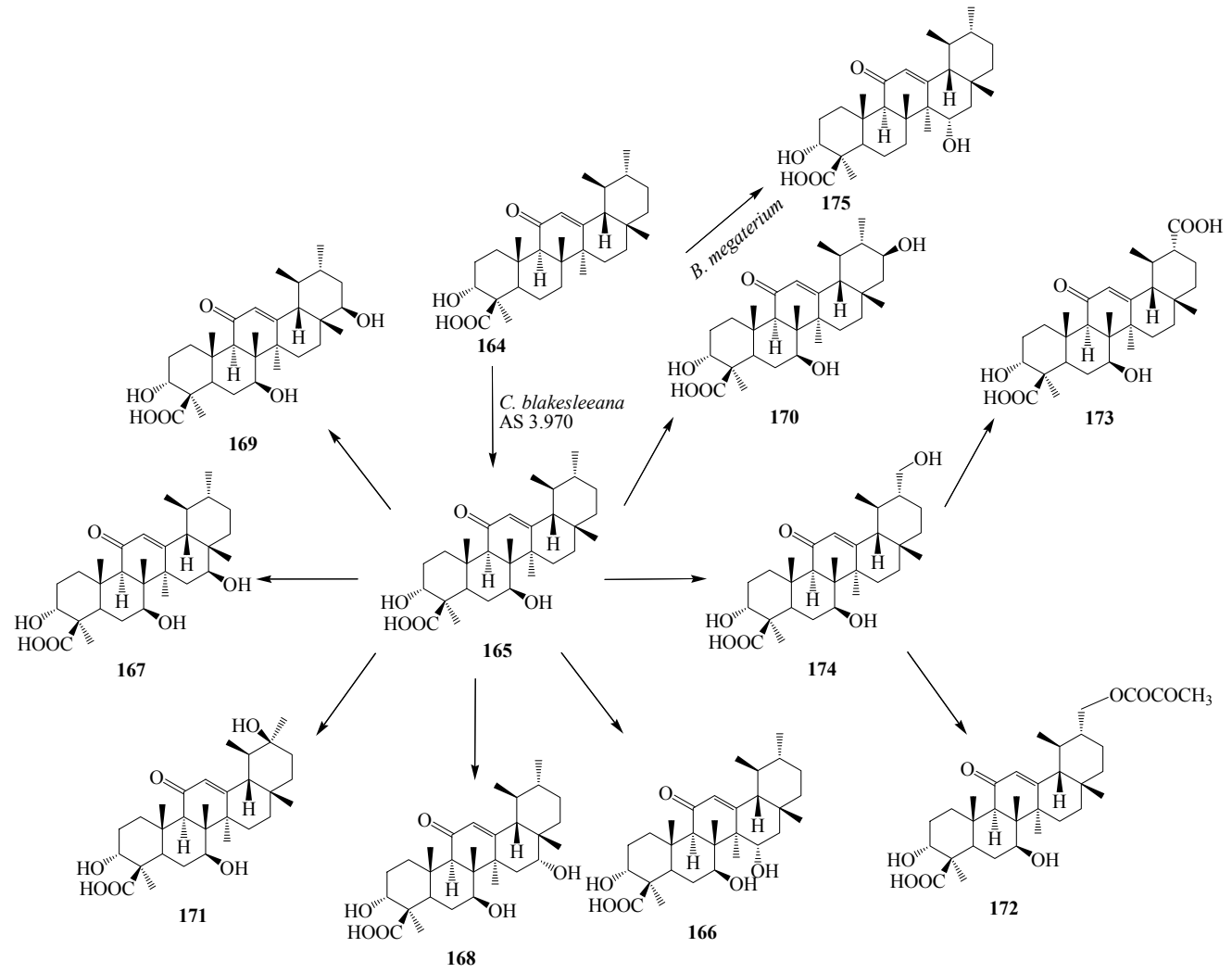

Microbial transformation of $\mathbf{1 7 6}$ by Nocardia corallina CGMCC4.1037 was reported by Feng et al. Incubation of $\mathbf{1 7 6}$ with $N$. corallina CGMCC4.1037 resulted three polar metabolites: 3-oxo-16 $\alpha$ hydroxy-olean-12-en-28-oic acid (177), 3 $\beta, 16 \alpha$-dihydroxy-olean-12-en-28-oic acid 28-O- $\beta$-Dglucopyranoside (178), and 3-oxo-16 $\alpha$-hydroxy-olean-12-en-28-oic acid 28- $O$ - $\beta$-D-glucopyranoside (179) as described in Figure 48 [63]. Wang et al. also reported the regio- and stereoselective modification of 176 by utlizing Rhizopus chinensis CICC 3043 and Alternaria alternata AS 3.4578 for lead for blocking HCV entry (see Figures 49 and 50) [64]. rThe major product from $R$. chinensis CICC 3043-mediated biotransformation was acacic acid lactone (180), along with five minor metabolites:

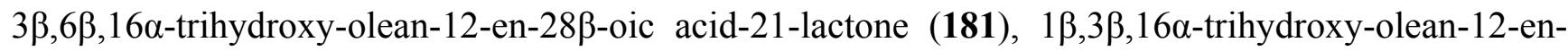
$28 \beta$-oic acid-21-lactone (182), 3 $\beta, 16 \alpha$-dihydroxy-olean-11,13(18)-dien-28 $\beta$-oic acid-21-lactone (183),

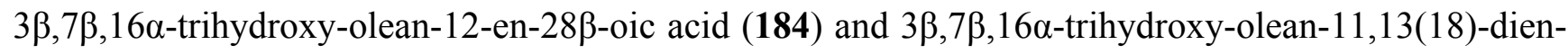
28ß-oic acid (185) (Figure 49). Furthermore, A. alternata AS 3.4578-mediated metabolism of 176 yielded two major metabolites identified as $1 \beta, 3 \beta, 16 \alpha$-trihydroxy-olean-11,13(18)-dien-28 $\beta$-oic acid (186) and 177, along with five minor metabolites: 183, 184, 185, 1 $\beta, 3 \beta, 16 \alpha$-trihydroxy-olean-12-en-

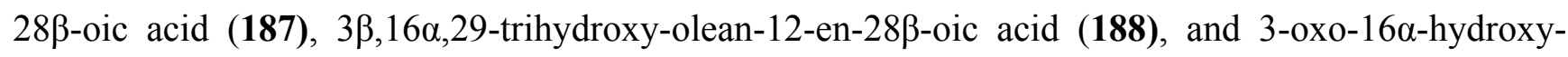
olean-12-en-28ß-oic acid (189) as presented in Figure $50[63,64]$. 
Figure 48. Microbial metabolism of echinocystic acid (176) with Nocardia corallina CGMCC4.1037.

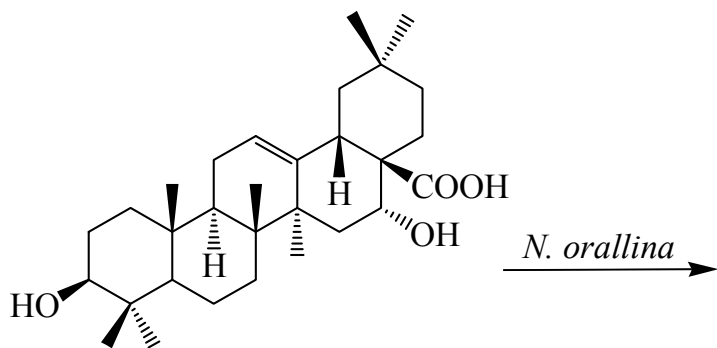

176

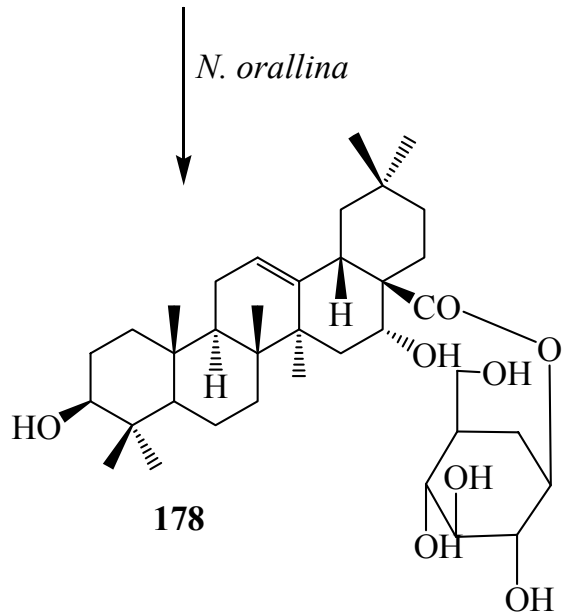

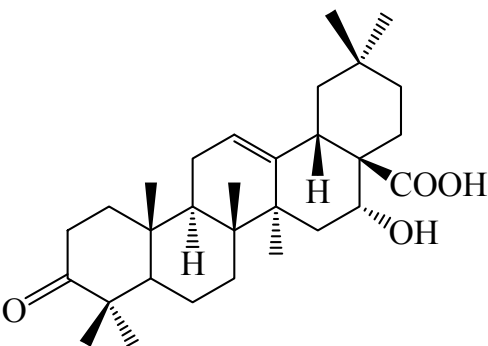

177

Figure 49. Microbial metabolism of echinocystic acid (176) with Rhizopus chinensis CICC 3043.

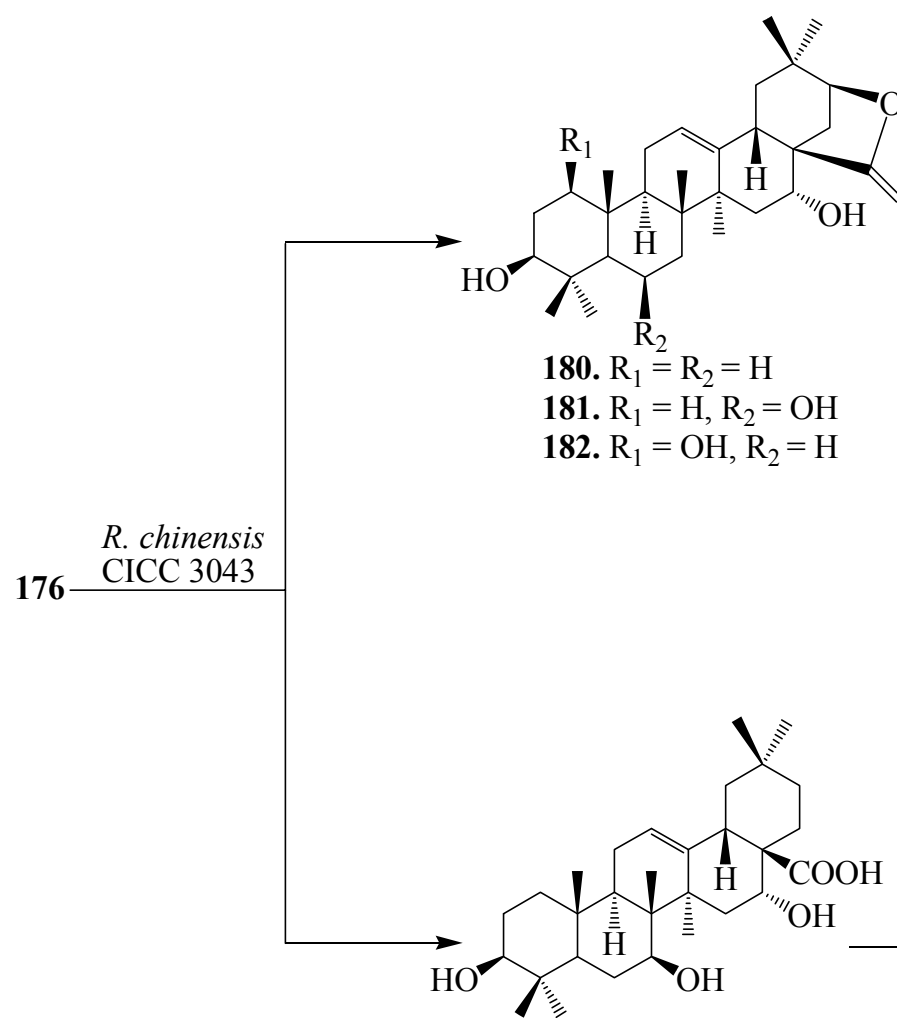

184

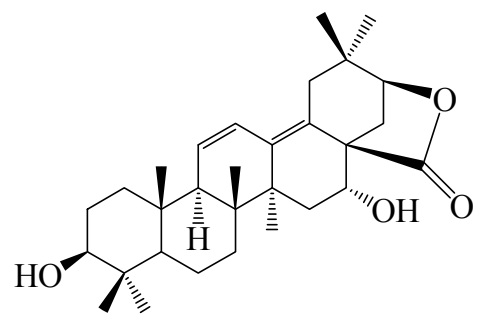

183

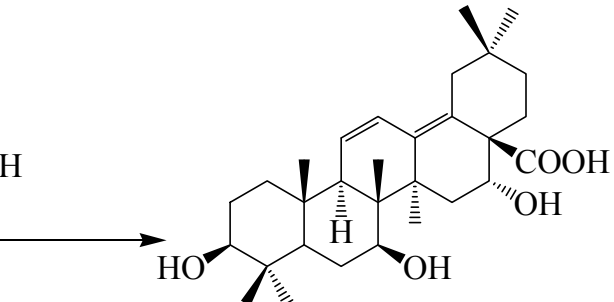

185 
Figure 50. Microbial metabolism of echinocystic acid (176) with Alternaria alternata AS 3.4578.

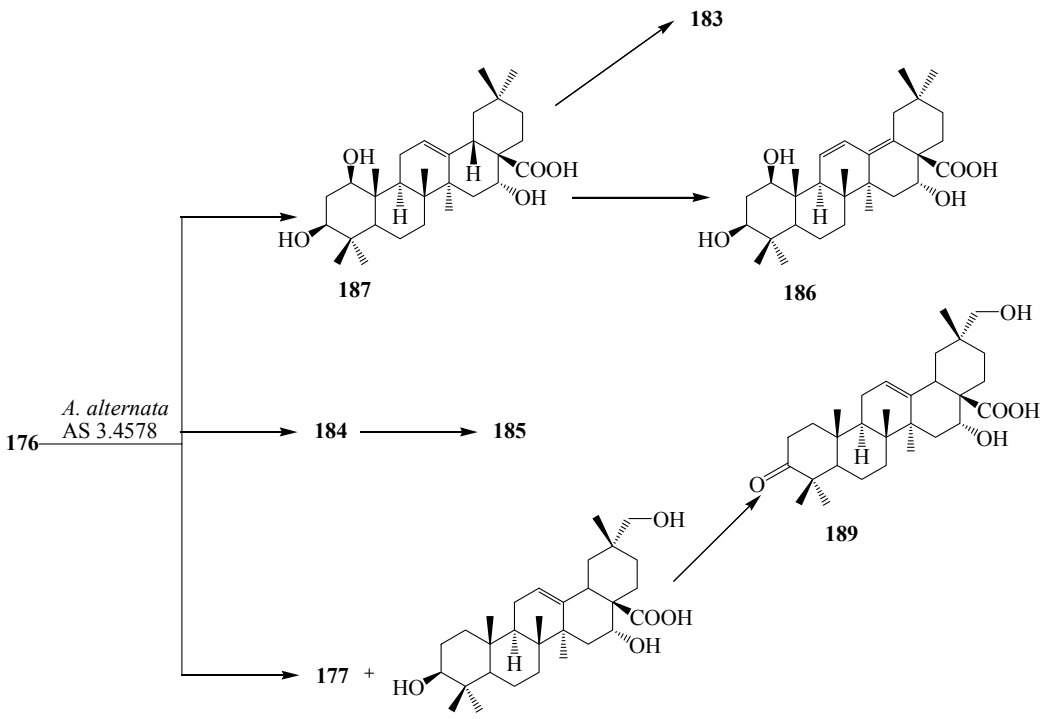

188

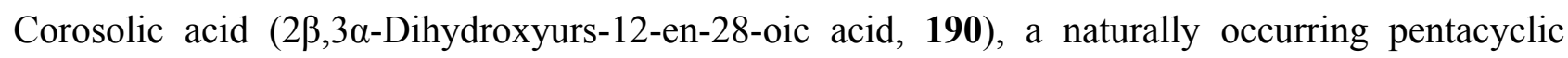
triterpene, has been found in many traditional Chinese medicinal herbs, such as Lagerstroemia speciosa, Eriobotrta japonica, Tiarella polyphylla, etc. It has also been found in variety of plants, such as in apples, basil, bilberries, cranberries, and prunes, and has been shown to have a number of biological activities, including suppression of cell proliferation and induction of apoptosis in various cancer cell lines [65,66]. The growing cultures of Fusarium equiseti CGMCC 3.3658 and Gliocladium catenulatum CGMCC 3.3655 were used for structural modification of corosolic acid (190) by Li et al. Incubation with $F$. equiseti CGMCC 3.3658 resulted two regioselective hydroxy metabolites $2 \alpha, 3 \beta, 15 \alpha$-trihydroxyurs-12-en-28-oic acid (191) and 2 $\alpha, 3 \beta, 7 \beta, 15 \alpha$-tetrahydroxyurs-12-en-28-oic acid (192) as shown in Figure 51. G. catenulatum CGMCC 3.3655 transformed 190 into $2 \alpha, 21 \beta$-dihydroxyA-homo-3 $\alpha$-oxours-12-en-28-oic acid (193), and 2 $\alpha, 3 \alpha, 21 \beta$-trihydroxyurs-12-en-28-oic acid (194) [67] (Figure 51).

Figure 51. Microbial transformation of corosolic acid (190) by Fusarium equiseti and Gliocladium catenulatum.

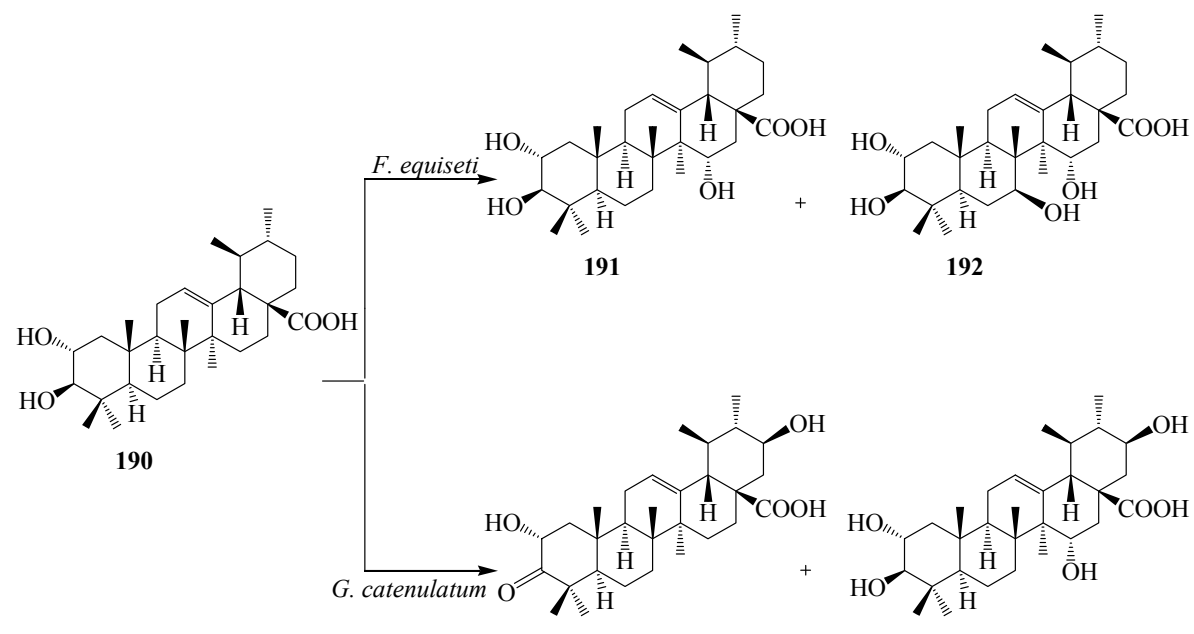

193 


\section{Concluding Remarks and Future Aspects}

In summary, microbial transformations are attractive alternative tools for the preparation of bioactive complex triterpenoids, which might be difficult to prepare by conventional chemical routes. They can produce commercially valuable pharmaceuticals for the biorefineries and novel lead molecules towards the development of new drug candidates. The transformation of triterpenoid skeleton through microorganisms in cell cultures exploited regioselective hydroxylations mainly in rings $\mathrm{A}, \mathrm{B}, \mathrm{C}, \mathrm{D}, \mathrm{E}$ and $\mathrm{C}-23, \mathrm{C}-24, \mathrm{C}-29$ and C-30 methyl groups, oxidation of C-28 methyl moiety and reduction of $\mathrm{C}-3$ alcohol group, ketones and $\mathrm{C}=\mathrm{C}$ bond at $\mathrm{C}-11$ and $\mathrm{C}-12$ positions. These modified triterpenoid drugs are currently favored when compared to their natural counterparts due to several therapeutic advantages. Moreover, microbial-catalyzed biotransformations in association with conventional organic synthesis can provide novel routes for the development of new drugs and drug candidates. A number of optimization techniques such as medium, temperature, agitation, $\mathrm{pH}$, etc., have to be established for microbial transformations to be successful and viable. Strain improvement by conventional methods or by genetic engineering identification of alternate biosynthetic routes via microorganisms that have not yet been exploited, new fermentation techniques and optimizing the production facilities will cut the manufacturing cost in future and allow the biotransformation processes to be more competitive to the current synthetic and isolation protocols.

\section{Acknowledgments}

Syed Adnan Ali Shah and Huey Ling Tan would like to acknowledge the Ministry of Higher Education (MOHE) for financial support under the Fundamental Research Grant Scheme (FRGS) with reference numbers 600-RMI/FRGS 5/3 (12/2012) and Research Acculturation Grant Scheme (RAGS) with reference number 600-RMI/RAGS 5/3 (21/2012). The authors would also like to acknowledge Universiti Teknologi MARA for the financial support under the Cumulative Impact Factor Initiative (CIFI) Grant Scheme with reference number UiTM 600-RMI/DANA 5/3/CIFI (117/2013) and the Principal Investigator Support Initiative (PSI) Grant Scheme with reference number UiTM 600RMI/DANA 5/3/PSI (251/2013).

\section{Author Contributions}

All authors equally contributed.

\section{Conflicts of Interest}

The authors declare no conflict of interest.

\section{References}

1. Salvador, J.A.R.; Moreira, V.M.; Gonçalves, B.M.F.; Lealab, A.S.; Jing, Y. Ursane-type pentacyclic triterpenoids as useful platforms to discover anticancer drugs. Nat. Prod. Rep. 2012, 29, 1463-1469.

2. Bauer, A.; Brönstrup, M. Industrial natural product chemistry for drug discovery and development. Nat. Prod. Rep. 2014, 31, 35-60. 
3. Newman, D.J.; Cragg, G.M. Natural products as sources of new drugs over the 30 years from 1981 to 2010. J. Nat. Prod. 2012, 75, 311-335.

4. Morrison, K.C.; Hergenrother, P.J. Natural products as starting points for the synthesis of complex and diverse compounds. Nat. Prod. Rep. 2014, 31, 6-14.

5. Bicas, J.L.; Dionìsio, A.P.; Pastore, G.M. Bio-oxidation of terpenes: An approach for the flavor industry. Chem. Rev. 2009, 109, 4518-4531.

6. Parshikov, I.A.; Netrusov, A.I.; Sutherland, J.B. Microbial transformation of antimalarial terpenoids. Biotechnol. Adv. 2012, 30, 1516-1523.

7. Parra, A.; Rivas, F.; Garcia-Granados, A.; Martinez, A. Microbial transformation of triterpenoids. Mini Rev. Org. Chem. 2009, 6, 307-320.

8. Muffler, K.; Leipold, D.; Schellera, M.; Haas, C.; Steingroewer, J.; Bley, T.; Neuhaus, H.E.; Mirata, M.A.; Schrader, J.; Ulber, R. Biotransformation of triterpenes. Process Biochem. 2011, 46, 1-15.

9. Shah, S.A.A.; Sultan, S.; Hassan, N.B.; Muhammad, F.K.B.; Faridz, M.A.B.M.; Hussain, F.B.M.; Hussain, M.; Adnan, H.S. Biotransformation of $17 \alpha$-ethynyl substituted steroidal drugs with microbial and plant cell cultures: A review. Steroids 2013, 78, 1312-1324.

10. Shah, S.A.A.; Sultan, S.; Zaimi, M. Biotransformation of tissue-specific hormone tibolone with fungal culture Trichothecium roseum. J. Mol. Struct. 2013, 1042, 118-122.

11. Shah, S.A.A.; Sultan, S.; Adnan, H.S. A Whole-cell biocatalysis application of steroidal drugs. Orient. J. Chem. 2013, 29, 389-403.

12. Azam, S.S.; Uddin, R.; Shah, S.A.A.; Zaheer-ul-Haq. Molecular docking studies of potent inhibitors of tyrosinase and $\alpha$-glucosidase. Med. Chem. Res. 2012, 21, 1677-1683.

13. Shah, S.A.A.; Sultan, S.; Adnan, H.S. Solid phase microbial transformation of cortexolone and prolyl endopeptidase inhibitory activity of the transformed products. Int. J. Pharm. Pharm. Sci. 2011, 3, 1-6.

14. Choudhary, M.I.; Shah, S.A.A.; Atta-ur-Rahman; Khan, S.N.; Khan, M.T.H. Alpha-glucosidase and tyrosinase inhibitors from fungal hydroxylation of tibolone. Steroids 2010, 75, 956-966.

15. Choudhary, M.I.; Shah, S.A.A.; Atta-ur-Rahman. Microbial transformation of anabolic steroids. Nat. Prod. Res. 2008, 22, 1289-1296.

16. Choudhary, M.I.; Batool, I.; Shah, S.A.A.; Khan, S.N.; Atta-ur-Rahman. Microbial transformation of oleanolic acid by Fusarium lini and $\alpha$-glucosidase inhibitory activity of its transformed products. Nat. Prod. Res. 2008, 22, 489-494.

17. Atta-ur-Rahman; Choudhary, M.I.; Basha, F.Z.; Abbas, G.; Khan, S.N.; Shah, S.A.A. Science at the interface of chemistry and biology: Discoveries of $\alpha$-glucosidase inhibitors and antiglycation agents. Pure Appl. Chem. 2007, 79, 2263-2267.

18. Choudhary, M.I.; Yousuf, S.; Samreen; Shah, S.A.A.; Ahmed, S.; Atta-ur-Rahman. Biotransformation of physalin $\mathrm{H}$ and antileishmanial activity of transformed product. Chem. Pharm. Bull. 2006, 54, 927-930.

19. Choudhary, M.I.; Shah, S.A.A.; Sami, A.; Ajaz, A.; Shaheen, F.; Atta-ur-Rahman. Fungal metabolites of $(E)$-guggulsterone and their antibacterial and radical-scavenging activities. Chem. Biodivers. 2005, 2, 516-524.

20. Choudhary, M.I.; Batool, I.; Shah, S.A.A.; Nawaz, S.A.; Atta-ur-Rahman. Microbial hydroxylation of pregnenolone derivative and cholinesterase inhibitory activity. Chem. Pharm. Bull. 2005, 53, $1455-1459$. 
21. Choudhary, M.I.; Shah, S.A.A.; Musharraf, S.G.; Shaheen, F.; Atta-ur-Rahman. Microbial transformation of dehydroepiandrosterone. Nat. Prod. Res. 2003, 17, 215-220.

22. Paludo, C.R.; da Silva-Junior, E.A.; Santos, R.A.; Pupo, M.T.; Emery, F.S.; Furtado, N.A.J.C. Microbial transformation of $\beta$-lapachone to its glycosides by Cunninghamella elegans ATCC 10028b. Phytochem. Lett. 2013, 6, 657-661.

23. Haldar, S.; Kolet, S.P.; Thulasiram, H.V. Biocatalysis: fungi mediated novel and selective 12 $\beta$ - or 17 $\beta$-hydroxylation on the basic limonoid skeleton. Green Chem. 2013, 15, 1311-1317.

24. Kuban, M.; Öngen, G.; Bedir, E. Biotransformation of cycloastragenol by Cunninghamella blakesleeana NRRL 1369 resulting in a novel framework. Org. Lett. 2010, 12, 4252-4255.

25. Kuban, M.; Öngen, G.; Khan, I.A.; Bedir, E. Microbial transformation of cycloastragenol. Phytochemistry 2013, 88, 99-104.

26. Yang, W.; Ye, M.; Huang, F.; He, W.; Guo, D. Biocatalysis of cycloastragenol by filamentous fungi to produce unexpected triterpenes. Adv. Synth. Catal. 2012, 354, 527-539.

27. Li, H.; Ye, M.; Guo, H.; Tian, Y.; Zhang, J.; Zhou, J.; Hub, Y.; Guo, D. Biotransformation of 20(S)-protopanaxadiol by Mucor spinosus. Phytochemistry 2009, 70, 1416-1420.

28. Chen, G.; Yang, X.; Li, J.; Ge, H.; Song, Y.; Ren, J. Biotransformation of 20(S)-protopanaxadiol by Aspergillus niger AS 3.1858. Fitoterapia 2013, 91, 256-260.

29. Schmitz, D.; Zapp, J.; Bernhardt, R. Hydroxylation of the triterpenoid dipterocarpol with CYP106A2 from Bacillus megaterium. FEBS J. 2012, 279, 1663-1674.

30. Sun, R.; Song, H.C.; Yang, Y.H.; Yang, P.; Yang, D.Y.; Shen, K.Z.; Xu, Y.B.; Gao, Y.X.; Chen, Y.G.; Dong, J.Y. Microbiological transformation of the triterpene nigranoic acid by the freshwater fungus Dictyosporium heptasporum. J. Asian Nat. Prod. Res. 2013, 15, 433-440.

31. Yang, Y.H.; Sun, R.; Song, H.C.; Xu, Y.B.; Yang, P.; Yang, D.Y.; Shen, Z.K.; Wanga, A.R.; Chen, Y.G.; Dong, J.Y. Microbial transformation of the triterpene nigranoic acid in Trichoderma sp. Phytochem. Lett. 2012, 5, 123-127.

32. Jäger, S.; Trojan, H.; Kopp, T.; Laszczyk, M.N.; Scheffler, A. Pentacyclic triterpene distribution in various plants-rich sources for a new group of multi-potent plant extracts. Molecules 2009, 14, 2016-2031.

33. Hua, S.; Ting, L.; Yan-Jun, S.; Li-Ming, Z.; Min, W. Preparation and crystal structure of 15 $\alpha$-hydroxyl-oleanolic acid. Chin. J. Struct. Chem. 2010, 29, 1798-1801.

34. Liu, D.L.; Liu, Y.; Qiu, F.; Gao, Y.; Zhang, J.Z. Biotransformation of oleanolic acid by Alternaria longipes and Penicillium Adametzi. J. Asian Nat. Prod. Res. 2011, 13, 160-167.

35. Capel, C.S.; de Souza, A.C.D.; de Carvalho, T.C.; de Sousa, J.P.B.; Ambrósio, S.R.; Martins, C.H.G.; Cunha, W.R.; Galán, R.H.; Furtado, N.A.J.C. Biotransformation using Mucor rouxii for the production of oleanolic acid derivatives and their antimicrobial activity against oral pathogens. J. Ind. Microbiol. Biotechnol. 2011, 38, 1493-1498.

36. Martinez, A.; Rivas, F.; Perojil, A.; Parra, A.; Garcia-Granados, A.; Fernandez-Vivas, A. Biotransformation of oleanolic and maslinic acids by Rhizomucor miehei. Phytochemistry 2013, 94, 229-237.

37. Gong, T.; Zheng, L.; Zhen, X.; He, H.; Zhu, H.; Zhu, P. Microbial transformation of oleanolic acid by Trichothecium roseum. J. Asian Nat. Prod. Res. 2014, 16, 383-386. 
38. Zhu, Y.Y.; Qian, L.W.; Zhang, J.; Liu, J.H.; Yu, B.Y. New approaches to the structural modification of olean-type pentacylic triterpenes via microbial oxidation and glycosylation. Tetrahedron 2011, 67, 4206-4211.

39. Guo, N.; Zhao, Y.; Fang, W. Biotransformation of 3-oxo-oleanolic acid by Absidia glauca. Planta Medica 2010, 76, 1904-1907.

40. Feng, X.; Luan, J.; Guo, F.F.; Li, D.P.; Chu, Z.Y. Microbial transformation of maslinic acid by Cunninghamella blakesleana. J. Mol. Catal. B Enzym. 2012, 82, 127-130.

41. Huang, F.; Yang, W.; Ye, F.; Tian, J.; Hu, H.; Feng, L.; Guo, D.; Ye, M. Microbial transformation of ursolic acid by Syncephalastrum racemosum (Cohn) Schroter AS 3.264. Phytochemistry 2012, $82,56-60$.

42. Fu, S.; Yang, J.; Cui, J.; Sun, D.A. Biotransformation of ursolic acid by Syncephalastrum racemosum CGMCC 3.2500 and anti-HCV activity. Fitoterapia 2013, 86, 123-128.

43. Nair, D.N.; Padmavathy, S. Impact of endophytic microorganisms on plants, environment and humans. Sci. World J. 2014, 1-11. doi:10.1155/2014/250693.

44. Fu, S.; Yang, J.; Cui, J.; Feng, X.; Sun, D.A. Biotransformation of ursolic acid by an endophytic fungus from medicinal plant Huperzia serrata. Chem. Pharm. Bull. 2011, 59, 1180-1182.

45. Leipold, D.; Wünscha, G.; Schmidt, M.; Bart, H.; Bley, T.; Neuhausd, H.E.; Bergmanne, H.; Richlinge, E.; Mufflera, K.; Ulbera, R. Biosynthesis of ursolic acid derivatives by microbial metabolism of ursolic acid with Nocardia sp. Strains-Proposal of new biosynthetic pathways. Process Biochem. 2010, 45, 1043-1051.

46. Ibrahim, A.; Khalifa, S.I.; Khafagi, I.; Youssef, D.T.; Khan, S.; Mesbah, M.; Khan, I. Microbial metabolism of biologically active secondary metabolites from Nerium oleander L. Chem Pharm Bull. 2008, 56, 1253-1258.

47. Fu, S.; Yang, J.; Cui, J.; Meng, Q.; Feng, X.; Sun, D. Multihydroxylation of ursolic acid by Pestalotiopsis microspora isolated from the medicinal plant Huperzia serrata. Fitoterapia 2011, 82, 1057-1061.

48. De Carvalho, T.C.; Polizeli, A.M.; Turatti, I.C.C.; de Carvalho M.E.S.C.E.; Ambrósio, S.R.; Crotti, A.E.M.; de Figueiredo, U.S.; Vieira, P.C.; Furtado, N.A.J.C. Screening of filamentous fungi to identify biocatalysts for lupeol biotransformation. Molecules 2010, 15, 6140-6151.

49. Yu-Juan, Q.; Bing, F.; Xin-Bo, S.; Wen-Bin, Z.; He-Shui, Y.; Li-Li, Z.; Li-Yan, Y.; Bai-Ping, M.A. Biotransformation of glycyrrhetinic acid by Cunninghamella blakesleeana. Chin. J. Nat. Med. 2010, 8, 373-381.

50. Xin, X.J.; Yang, G.; Gou, Z.P.; Yao, J.H.; Lana, R.; Ma, X.C. Structural determination of two newtriterpenoids biotransformed from glycyrrhetinic acid by Mucor polymorphosporus. Magn. Reson. Chem. 2010, 48, 164-167.

51. Maatooq, G.T.; Marzouk, A.M.; Gray, A.I.; Rosazza, J.P. Bioactive microbial metabolites from glycyrrhetinic acid. Phytochemistry 2010, 71, 262-270.

52. Qi-he, C.; Jing, L.; Hai-feng, Z.; Guo-qing, H.; Ming-liang, F. The betulinic acid production from betulin through biotransformation by fungi. Enzym. Microb. Technol. 2009, 45, 175-180.

53. Feng, Y.; Li, M.; Liu, J.; Xu, T.Y.; Fang, R.S.; Chen, Q.H.; He, G.Q. A novel one-step microbial transformation of betulin to betulinic acid catalysed by Cunninghamella blakesleeana. Food Chem. 2013, 136, 73-79. 
54. Grishko, V.V.; Tarasova, E.V.; Ivshina, I.B. Biotransformation of betulin to betulone by growing and resting cells of the actinobacterium Rhodococcus rhodochrous IEGM 66. Process Biochem. 2013, 48, 1640-1644.

55. Mao, D.B.; Feng, Y.Q.; Bai, Y.H.; Xu, C.P. Novel biotransformation of betulin to produce betulone by Rhodotorula Mucilaginosa. J. Taiwan Inst. Chem. Eng. 2012, 43, 825-829.

56. Liu, H.; Lei, X.L.; Li, N.; Zong, M.H. Highly regioselective synthesis of betulone from betulin by growing cultures of marine fungus Dothideomycete sp. HQ 316564. J. Mol. Catal. B Enzym. 2013, 88, 32-35.

57. Baratto, L.C.; Porsani, M.V.; Pimentel, I.C.; Netto, A.B.P.; Paschke, R.; Oliveira, B.H. Preparation of betulinic acid derivatives by chemical and biotransformation methods and determination of cytotoxicity against selected cancer cell lines. Eur. J. Med. Chem. 2013, 68, 121-131.

58. Zhang, J.; Cheng, Z.H.; Yu, B.Y.; Cordell, G.A.; Qiu, S.X. Novel biotransformation of pentacyclic triterpenoids acids by Nocardia sp. NRRL 5646. Tetrahedron Lett. 2005, 46, 2337-2340.

59. Qian, L.W.; Zhang, J.; Liu, J.H.; Yu, B.Y. Direct microbial-catalyzed asymmetric $\alpha$-hydroxylation of betulonic acid by Nocardia sp. NRRL 5646. Tetrahedron Lett. 2009, 50, 2193-2195.

60. Qian, L.; Zhang, J.; Liu, J.; Yu, B. Regio-specific microbial hydroxylation of phytolaccagenin by Streptomyces griseus ATCC 13273. Chin. J. Chem. 2009, 27, 1434-1436.

61. Wang, Y.; Sun, Y.; Wang, C.; Huo, X.; Liu, P.; Wang, C.; Zhang, B.; Zhan, L.; Zhang, H.; Deng, S.; et al. Biotransformation of 11-keto-b-boswellic acid by Cunninghamella blakesleana. Phytochemistry 2013, 96, 330-336.

62. Bleif, S.; Hannemann, F.; Zapp, J.; Hartmann, D.; Jauch, J.; Bernhardt, R. A new Bacillus megaterium whole-cell catalyst for the hydroxylation of the pentacyclic triterpene 11-keto-beta-boswellic acid (KBA) based on a recombinant cytochrome P450 system. Appl. Microbiol. Biotechnol. 2012, doi:10.1007/s00253-011-3467-0.

63. Feng, X.; Zou, Z.; Fu, S.; Sun, L.; Su, Z.; Sun, D. Microbial oxidation and glucosidation of echinocystic acid by Nocardia coralline. J. Mol. Catal. B Enzym. 2010, 66, 219-223.

64. Wang, H.; Wang, Q.; Xiao, S.; Yu, F.; Ye, M.; Zheng, Y.X.; Zhao, C.K.; Sun, D.; Zhang, L.H.; Zhou, D.M. Elucidation of the pharmacophore of echinocystic acid, a new lead for blocking HCV entry. Eur. J. Med. Chem. 2013, 64, 160-168.

65. Nho, K.J.; Chun, J.M.; Kim, H.K. Corosolic acid induces apoptotic cell death in human lung adenocarcinoma A549 cells in vitro. Food Chem. Toxicol. 2013, 56, 8-17.

66. Kim, J.; Kim, Y.H.; Song, G.; Kim, D.; Jeong, Y.; Liu, K.; Chung, Y.; Oh, S. Ursolic acid and its natural derivative corosolic acid suppress the proliferation of APC-mutated colon cancer cells through promotion of $\beta$-catenin degradation. Food Chem. Toxicol. 2014, 67, 87-95.

67. Li, P.; Feng, X.; Chu, Z.; Guo, F.; Zhang, Z. Microbial transformation of corosolic acid by Fusarium equiseti and Gliocladium catenulatum. J. Asian Nat. Prod. Res. 2013, 15, 789-808.

(C) 2014 by the authors; licensee MDPI, Basel, Switzerland. This article is an open access article distributed under the terms and conditions of the Creative Commons Attribution license (http://creativecommons.org/licenses/by/3.0/). 